\title{
Rocky Planet Formation: Quick and Neat
}

\author{
Scott J. Kenyon \\ Smithsonian Astrophysical Observatory, 60 Garden Street, Cambridge, MA 02138 USA \\ Joan R. Najita \\ National Optical Astronomy Observatory, 950 Cherry Avenue, Tucson, AZ. 85719, USA \\ Benjamin C. Bromley \\ Department of Physics 63 Astronomy, University of Utah, 201 JFB, Salt Lake City, UT \\ 84112 USA
}

\begin{abstract}
We reconsider the commonly held assumption that warm debris disks are tracers of terrestrial planet formation. The high occurrence rate inferred for Earthmass planets around mature solar-type stars based on exoplanet surveys $(\sim 20 \%)$ stands in stark contrast to the low incidence rate $(\leq 2 \%-3 \%)$ of warm dusty debris around solar-type stars during the expected epoch of terrestrial planet assembly $(\sim 10 \mathrm{Myr})$. If Earth-mass planets at AU distances are a common outcome of the planet formation process, this discrepancy suggests that rocky planet formation occurs more quickly and/or is much neater than traditionally believed, leaving behind little in the way of a dust signature. Alternatively, the incidence rate of terrestrial planets has been overestimated or some previously unrecognized physical mechanism removes warm dust efficiently from the terrestrial planet region. A promising removal mechanism is gas drag in a residual gaseous disk with a surface density $\gtrsim 10^{-5}$ of the minimum mass solar nebula.
\end{abstract}

Subject headings: planetary systems - planets and satellites: formation - protoplanetary disks - stars: formation - circumstellar matter

\section{Introduction}

The conventional picture of terrestrial planet formation begins with the growth of 1$10 \mathrm{~mm}$ pebbles from $1 \mu \mathrm{m}$ dust grains (Chiang \& Youdin 2010; Birnstiel et al. 2010; Youdin 2010; Windmark et al. 2012; Garaud et al. 2013; Birnstiel et al. 2016). Collisional and 
collective processes then convert pebbles into km-sized or larger planetesimals (Youdin \& Goodman 2005; Johansen et al. 2007; Youdin 2011a; Johansen et al. 2015; Simon et al. 2016).

Various observations support the idea that planetesimals in protoplanetary disks grow rapidly (within $\sim 1 \mathrm{Myr}$ ), including (i) radiometric analyses of meteorites (Bizzarro et al. 2005; Kleine et al. 2009; Schulz et al. 2009; Dauphas \& Chaussidon 2011; Dauphas \& Pourmand 2011; Sugiura \& Fujiya 2014), (ii) comparisons of the mass distributions of solids in protostellar disks and known exoplanet populations (Najita \& Kenyon 2014), (iii) the trend in the abundance of $\mathrm{HCN}$ relative to $\mathrm{H}_{2} \mathrm{O}$ as a function of disk mass (Najita et al. 2013), and (iv) coherent structure in the young HL Tau disk, as observed by ALMA (ALMA Partnership et al. 2015; Zhang et al. 2015).

Once planetesimals form, they quickly $\left(10^{4}-10^{5} \mathrm{yr}\right)$ merge into much larger protoplanets (Weidenschilling 1974; Wetherill 1980). Over 1-100 Myr, a series of giant impacts among the protoplanets then leads to several Earth-mass planets (Chambers \& Wetherill 1998; Chambers 2001; Raymond et al. 2004; Kenyon \& Bromley 2006; Lunine et al. 2011; Raymond et al. 2014). Throughout this period, theory predicts a disk-shaped cloud of collisional debris that produces an observable infrared (IR) excess. These debris disks are expected to serve as signposts of ongoing rocky planet formation (e.g., Kenyon \& Bromley 2002; Zuckerman \& Song 2004; Kenyon \& Bromley 2004; Raymond et al. 2011, 2012; Leinhardt et al. 2015).

If warm debris is a dependable beacon, it provides a simple way to locate sites of ongoing terrestrial planet formation and to measure the frequency with which rocky planetary systems form. Compared to the challenges of identifying Earth-mass planets from direct imaging, microlensing, radial velocity, and transit observations (e.g., Gould et al. 2006; Cumming et al. 2008; Macintosh et al. 2014; Burke et al. 2015), measuring the magnitude of an IR excess is fairly straightforward and independent of viewing geometry (e.g., Carpenter et al. 2009b a; Kennedy \& Wyatt 2013; Patel et al. 2014). IR observations are often sufficient to establish the temperature and location of the debris (e.g., Lisse et al. 2008; Currie et al. 2011). Theoretical models then allow us to use this information to construct a window into the planet formation process (e.g., Genda et al. 2015b; Kenyon \& Bromley 2016).

Here, we examine the reliability of warm debris disks as tracers of ongoing terrestrial planet formation. Current observations suggest the frequency of warm debris disks around young solar-type stars $(\$ 2)$ is much smaller than the frequency of Earth-mass planets around older solar-type stars $(\$ 3)$. Analytical calculations of dust emission during the final phases of planet assembly indicate that all popular scenarios of rocky planet formation predict detectable amounts of debris for stellar ages of 5-20 Myr (\$4). Thus, there is a clear discrepancy between theoretical predictions and the observed frequencies of warm debris disks and Earth-mass planets. 
In \$5, we consider options for resolving this discrepancy. After constraining uncertainties in the frequency of Earth-mass planets (\$5.1), dust emission (\$5.2), and theoretical predictions $(\$ 5.3)$, we demonstrate that if stars retain a residual gaseous disk with a surface density $\gtrsim 10^{-5}$ of the minimum mass solar nebula (\$5.4), gas drag and radiation pressure will rapidly remove small grains from the terrestrial planet region. This mechanism is plausible given current observational limits on gaseous disks among 5-20 Myr old stars (\$5.5), We conclude with a set of suggestions to test the possibilities for reconciling theory and observations ( $(5.6)$ and a brief summary $(\$ 6)$.

\section{WARM EXCESSES FROM DEBRIS DISKS ARE RARE}

Although opaque protoplanetary disks surround essentially all newly-formed stars (e.g., Kenyon et al. 2008; Williams \& Cieza 2011; Andrews 2015, and references therein), the infrared excess emission produced by the disk vanishes roughly simultaneously at all wavelengths on a time scale of $\sim 3$ Myr (Haisch et al. 2001; Kennedy \& Kenyon 2009; Mamajek 2009; Williams \& Cieza 2011; Alexander et al. 2014). The ultraviolet excess produced by accretion onto the central star declines on a similar time scale (Hartmann et al. 1998; Kennedy \& Kenyon 2009, Sicilia-Aguilar et al. 2010; Ingleby et al. 2014).

Among older, solar-type main sequence stars, roughly $20 \%$ have optically thin IR or mm emission from debris disks (e.g., Hillenbrand et al. 2008; Trilling et al. 2008; Eiroa et al. 2013). Typically, this emission is comparable to or less than the stellar flux at 8-25 $\mu \mathrm{m}$ and a factor of $\gtrsim 10$ larger than the stellar flux at longer wavelengths. To relate excesses to a fractional luminosity, we assume the emission arises from a single-temperature, optically thin dust component with temperature $T_{d}$ located at a distance $a$ from a star with luminosity $L_{\star}$, mass $M_{\star}$, radius $R_{\star}$, and temperature $T_{\star}$ :

$$
\left(\frac{T_{d}}{T_{\star}}\right)^{4} \approx\left(\frac{R_{\star}}{2 a}\right)^{2} .
$$

The dust to star luminosity ratio is

$$
\frac{L_{d}}{L_{\star}}=\left(\frac{T_{d}}{T_{\star}}\right)^{4} \frac{A_{d}}{\pi R_{\star}^{2}},
$$

where $A_{d}$ is the total cross-sectional area of all solid particles. Assuming the grains emit as blackbodies, we can relate $L_{d} / L_{\star}$ to the flux ratio at any wavelength:

$$
\frac{L_{d}}{L_{\star}}=\frac{\left(e^{h \nu / k T_{d}}-1\right)}{\left(e^{h \nu / k T_{\star}}-1\right)}\left(\frac{T_{d}}{T_{\star}}\right)^{4} \frac{F_{d}}{F_{\star}} .
$$


For $T_{d} \approx 280 \mathrm{~K}, T_{\star} \approx 5800 \mathrm{~K}$, and $F_{d} / F_{\star} \lesssim 1$ at $12 \mu \mathrm{m}(24 \mu \mathrm{m}), L_{d} / L_{\star} \lesssim 10^{-3}\left(10^{-4}\right)$. Debris disks are a factor $\gtrsim 100-1000$ less luminous than the protoplanetary disks surrounding T Tauri stars (see also Wyatt 2008; Carpenter et al. 2009b).

Fig. 1 illustrates the variation of $F_{d}(a) / F_{\star}$ at $8-24 \mu \mathrm{m}$ for $L_{d} / L_{\star}=10^{-3}$ (violet curves) and $10^{-4}$ (orange curves). Adopting detection limits from Spitzer $(8 \mu \mathrm{m}, 16 \mu \mathrm{m}, 24 \mu \mathrm{m}$; Carpenter et al. 2009b) and WISE (12 $\mu \mathrm{m} ;$ Luhman \& Mamajek 2012), $F_{d} / F_{\star} \approx 0.03-0.25$, it is clear that detecting an excess at 8-12 $\mu \mathrm{m}$ from dust at $\sim 1 \mathrm{AU}$ requires $L_{d} / L_{\star} \gtrsim$ $3 \times 10^{-4}$. At longer wavelengths, it is possible to detect warm dust with a substantially lower luminosity, $L_{d} / L_{\star} \gtrsim 3 \times 10^{-5}$ to $10^{-4}$. Because "cold" dust $\left(T_{d} \lesssim 200 \mathrm{~K}\right)$ from beyond the terrestrial planet region $(>1-2 \mathrm{AU})$ can also contribute to the observed $24 \mu \mathrm{m}$ emission, observations at shorter wavelengths (e.g., 8-16 $\mu \mathrm{m}$ ) or at longer wavelengths (e.g., $70 \mu \mathrm{m})$ are required to constrain $T_{d}$ and $L_{d} / L_{\star}$.

To put an observed $L_{d} / L_{\star}$ in perspective, we derive the required mass $M_{d}$ in solid particles. In standard models, the solids have a power-law size distribution, $N(r) \propto r^{-q}$ where $r$ is the radius of a particle and $q \approx 3.5$. Thus, $M_{d} \approx(4 / 3) \rho A_{d}\left(r_{\min } r_{\max }\right)^{1 / 2}$, where $\rho$ is the mass density, $r_{\min }$ is the radius of the smallest particle, and $r_{\max }$ is the mass of the largest particle (Wyatt 2008). For material with $r_{\min }=1 \mu \mathrm{m}, r_{\max }=300 \mathrm{~km}$, and $L_{d} / L_{\star}=10^{-4}$ at $r=1 \mathrm{AU}, M_{d} \approx 3 \times 10^{25} \mathrm{~g}$. Thus, an observable excess at $24 \mu \mathrm{m}$ requires roughly a third of a lunar mass of solid material at 1 AU.

Over the past decade, various surveys suggest a very low frequency of warm debris disks among solar-type stars (e.g., Stauffer et al. 2005; Silverstone et al. 2006; Currie et al. 2007; Carpenter et al. 2009b a; Chen et al. 2011; Luhman \& Mamajek 2012; Kennedy \& Wyatt 2013; Cloutier et al. 2014; Matthews et al. 2014, and references therein). To illustrate current constraints on the incidence rate of warm dust, we describe in detail several studies using data from Spitzer and WISE.

The Spitzer Formation and Evolution of Planetary Systems (FEPS) program surveyed 314 solar-type stars at 3-70 $\mu \mathrm{m}$ (Meyer et al. 2006; Carpenter et al. 2009b). The sample included stars in clusters and the field, with ages ranging from 3 Myr to 3 Gyr. Within this group, only 5 have a measurable $16 \mu \mathrm{m}$ excess. All five stars also have substantial excess emission at $8 \mu \mathrm{m}, 24 \mu \mathrm{m}$, and 60-100 $\mu \mathrm{m}$ and prominent accretion signatures from hot gas close to the central star. Thus, all are primordial disks, the gas-rich disks commonly observed in T Tauri stars (Silverstone et al. 2006; Dahm \& Carpenter 2009; Carpenter et al. 2009b).

All of the non-primordial disk excess sources have the modest dust luminosities, $L_{d} / L_{\star} \lesssim$ $10^{-3}$, characteristic of debris disks. However, few if any sources have obvious emission from 
warm dust within $1 \mathrm{AU}$ of the star (Carpenter et al. 2009b). Excluding the primordial disks, no source has an excess at $8 \mu \mathrm{m}(16 \mu \mathrm{m})$ above $3 \%(16 \%)$ of the stellar photosphere. From $R_{24 / 8}$, the ratio of the $24 \mu \mathrm{m}$ to the $8 \mu \mathrm{m}$ flux, $16 \%$ of all FEPS sources have robust detections of $24 \mu \mathrm{m}$ dust emission $\gtrsim 10 \%$ above the stellar photosphere. Among the 30 stars younger than $10 \mathrm{Myr}, 3 \%, 7 \%$, and $20 \%$ of the non-primordial disk sources have a detected $24 \mu \mathrm{m}$ excess at 50\%,30\%, and 10\% above the stellar photosphere.

Detailed fits of model spectral energy distributions for non-blackbody grains to the Spitzer IRS spectra at 8-35 $\mu \mathrm{m}$ for excess sources of all ages yield average inferred dust temperatures $T_{d} \approx 45 \mathrm{~K}$ to $200 \mathrm{~K}$ with a median at $112 \mathrm{~K}$. Typical inner disk radii range from $a_{\text {in }} \approx 2-3$ AU to $40 \mathrm{AU}$ with a median at $6 \mathrm{AU}$. Adopting a blackbody model for dust emission yields similar median values for $T_{d}$ and $a_{\text {in }}$, with somewhat larger ranges $\left(T_{d} \approx\right.$ 50-280 K and $a_{\mathrm{in}} \approx 1-31 \mathrm{AU}$ ). Only a few (between 1 and 3) $24 \mu \mathrm{m}$ excess sources have $T_{d} \gtrsim 200 \mathrm{~K}$ and $a_{\text {in }} \approx 1-2 \mathrm{AU}$, demonstrating that there is little obvious evidence for warm dust in the terrestrial zones of FEPS targets. The excess limits at wavelengths shortward of $24 \mu \mathrm{m}$ constrain the fraction of the $24 \mu \mathrm{m}$ excess that could be produced by warm dust (\$4).

Although the FEPS study has a high sensitivity to IR excess as a fraction of the stellar photosphere, it covers a wide range in stellar age and has a relatively small $(\sim 30)$ set of sources with ages $(\lesssim 10 \mathrm{Myr})$ relevant to terrestrial planet assembly. To complement these results, we examine the Spitzer and WISE statistics for the well-studied Upper Scorpius association (Upper Sco). Upper Sco, which is part of the nearby Sco-Cen association (Sco OB2; Preibisch \& Mamajek 2008), allows an accurate census of dust emission at ages of 712 Myr (Luhman \& Mamajek 2012; Pecaut et al. 2012; Rizzuto et al. 2016), when terrestrial planets accumulate most of their final mass (e.g., Dauphas \& Chaussidon 2011; Raymond et al. 2014; Quintana et al. 2016).

Analyses of Spitzer data alone indicate a very small frequency of warm debris disks among solar-type stars in Upper Sco (Carpenter et al. 2006; Dahm \& Carpenter 2009; Carpenter et al. 2009a; Chen et al. 2011). In a sample of 27 K-type pre-main sequence stars with masses of $0.8-1.3 M_{\odot}$, most of the excess sources $(7 / 9)$ have IR colors consistent with primordial disks (Carpenter et al. 2006, 2009a). Although two additional stars have $24 \mu \mathrm{m}$ excesses consistent with debris disks, both have cool dust $\left(T_{d} \lesssim 200 \mathrm{~K}\right)$. Within a much larger sample of 101 M-type stars with masses smaller than $0.8 M_{\odot}, 9(17)$ are debris (primordial) disks. The lack of short wavelength excesses among stars with debris disks suggest none of these have substantial amounts of warm dust.

To enlarge the sample of solar-type stars, Luhman \& Mamajek (2012) added WISE data to previous Spitzer surveys. Among K0-M0 stars with masses of $0.7-1.3 M_{\odot}, 28 \%(17 / 60)$ have a $22-24 \mu \mathrm{m}$ excess and $13 \%(9 / 68)$ have a $12 \mu \mathrm{m}$ excess at levels $\gtrsim 25 \%$ of the stellar 
photosphere. There is little evidence for warm debris. All of the $12 \mu \mathrm{m}$ excess sources are classified as "full, transitional, or evolved disks"; none are debris disks. When all detection statistics are considered (Luhman \& Mamajek 2012), fewer than 3\% of Upper Sco sources have a $12 \mu \mathrm{m}$ excess from a debris disk. Among the $22-24 \mu \mathrm{m}$ excess sources without $8 \mu \mathrm{m}$ or $12 \mu \mathrm{m}$ excesses, eight are classified as "debris or evolved transitional" disks. For the two stars with large $22-24 \mu \mathrm{m}$ excesses, the lack of an accompanying $8 \mu \mathrm{m}$ or $16 \mu \mathrm{m}$ excess indicates that most of the dust is cold. The maximum frequency of warm debris disks in this sample is then $10 \%(6 / 60)$.

Although there are no constraints on dust temperature for the other six stars, if the FEPS results are a guide, most contain cold dust. Among FEPS sources with $24 \mu$ m excesses that overlap in age with Upper Sco (5-20 Myr), roughly $80 \%$ have cold dust (Carpenter et al. 2009b). Adopting this scaling for Upper Sco, only 1-2 of the six 22-24 $\mu \mathrm{m}$ excess sources contain warm dust. Thus, a more realistic estimate for the fraction of 10 Myr old stars with warm dust is $\sim 2 \%$.

Many other studies also conclude that warm excesses from debris disks around solar-type stars are extremely rare (e.g., Moór et al. 2009; Stauffer et al. 2010; Beichman et al. 2011; Smith et al. 2011; Zuckerman et al. 2011; Ribas et al. 2012; Urban et al. 2012; Zuckerman et al. 2012; Jackson \& Wyatt 2012; Kennedy \& Wyatt 2012; Ballering et al. 2013; Vican \& Schneider 2014). Although IRAS, Spitzer, and Herschel data suggest $10 \%$ to $30 \%$ of solartype main sequence stars have IR excesses (e.g., Lagrange et al. 2000; Eiroa et al. 2013, Ballering et al. 2013, and references therein), nearly all sources have color temperatures characteristic of cold dust $\left(T_{d} \ll 300 \mathrm{~K}\right)$ at $a \gg 1 \mathrm{AU}$. In addition to Upper Sco, Spitzer data for the young (15 Myr) clusters $h$ and $\chi$ Per suggest a small excess fraction of $1 \%$ to $2 \%$ at $8 \mu \mathrm{m}$ (Currie et al. 2007; Cloutier et al. 2014). Analyses of WISE data for stars in the solar neighborhood yield similarly small $(\lesssim 1 \%-2 \%)$ fractions of sources with a $12 \mu \mathrm{m}$ excess (Kennedy \& Wyatt 2013; Patel et al. 2014).

\section{EARTH MASS PLANETS AT 0.25-1 AU ARE FAIRLY COMMON}

In contrast to the small fraction of stars with warm debris disks, rocky planets within 1 AU appear to be fairly common companions to solar-type stars (Youdin 2011b; Fang \& \begin{tabular}{l|l|l|l|} 
Margot 2012; & Batalha et al. 2013; Foreman-Mackey et al. 2014; & Silburt et al. 2015; Winn \& \\
\hline
\end{tabular} Fabrycky 2015). Although the false positive rate is uncertain, recent attempts to confirm Kepler candidates with ground-based and other space-based observations suggest false positive rates ranging from $\sim 10 \%$ to $75 \%$ for various ranges of planet masses (e.g., Morton \& Johnson 2011; Santerne et al. 2012; Fressin et al. 2013; Sliski \& Kipping 2014; Désert et al. 
2015; Colón et al. 2015; Santerne et al. 2016; Coughlin et al. 2016; Mullally et al. 2016; Morton et al. 2016). Here, we focus on a comprehensive analysis of Kepler data which provides a detailed estimate for the occurrence rate of Earth-mass planets inside $1 \mathrm{AU}$. This approach is conservative: the formation of lower- and higher-mass rocky planets also produces observable amounts of debris. Assuming false positive rates are small, these analyses thus yield robust lower limits to the fraction of solar-type stars that produce detectable debris at ages of 5-20 Myr. We return to the false positive rate in $\S 5$.

Using Q1-Q16 Kepler data, Burke et al. (2015) estimate 0.77 planets with radii of 0.7-2.5 $\mathrm{R}_{\oplus}$ and orbital periods of 50-300 days ( 0.25-0.9 AU) per GK dwarf star (see also Petigura et al. 2013; Mullally et al. 2015, Coughlin et al. 2016, and references therein). Recent detailed analyses of transiting planets with radial velocity measurements suggest most planets with radii smaller than 1.5-2 $\mathrm{R}_{\oplus}$ have rocky compositions (e.g., Weiss \& Marcy 2014; Marcy et al. 2014; Buchhave et al. 2014). Applying these results to their complete Kepler samples, Burke et al. (2015) derive a probability of 0.1 for an Earth-mass planet $\left(0.8-1.2 \mathrm{R}_{\oplus}\right)$ at $0.86-1.13$ $\mathrm{AU}$ and a probability of 0.075 for an Earth-mass planet $\left(0.8-1.2 \mathrm{R}_{\oplus}\right)$ at $0.61-0.8 \mathrm{AU}$. Taken at face value, these rates predict a somewhat larger frequency of planets (per unit area) at $0.7 \mathrm{AU}$ than at $1 \mathrm{AU}$. Integrating over 0.6-1 AU, the probability is roughly 0.19 (assuming an intermediate rate for the missing region at 0.8-0.86 AU). Although Burke et al. (2015) do not quote a rate for Earth-mass planets within 0.25-0.6 AU, plausible extrapolations of their rates yield a probability of $0.22-0.25(0.21-0.23)$ for an Earth mass planet $\left(0.8-1.2 \mathrm{R}_{\oplus}\right)$ within $0.25-1$ AU (0.4-1 AU) of a solar-type star.

Unless the false positive rate for the Kepler sample of Earth-mass planet candidates is much larger than $50 \%$, the roughly $20 \%$ incidence rate for rocky planets with radii of 0.8-1.2 $\mathrm{R}_{\oplus}$ at $0.25-1 \mathrm{AU}$ is much larger than the $\lesssim 2-3 \%$ rate of warm debris disks among solar-type stars with ages of 5-30 Myr. The discrepancy between the apparent formation rate of terrestrial planets and the detection rate for terrestrial debris disks is probably much larger than suggested by these estimates. We anticipate considerable debris from (i) the formation of rocky planets smaller than $0.8 \mathrm{R}_{\oplus}$ and larger than $1.2 \mathrm{R}_{\oplus}$ at $0.25-1 \mathrm{AU}$ and (ii) the formation of any rocky planet at $\lesssim 0.25 \mathrm{AU}$ and at $1-2 \mathrm{AU}$. If these formation channels yield a substantial population of rocky planets, the detection rate for debris disks is at least a factor of ten smaller than expected from the incidence rates of rocky planets.

\section{DEBRIS GENERATION FROM PLANET FORMATION}

To assess the significance of the different detection rates for warm debris disks and Earthmass planets inside $2 \mathrm{AU}$, we must estimate the amount and lifetime of debris produced by 
terrestrial planet formation. To make this evaluation, we rely on theoretical estimates from popular scenarios that produce terrestrial planets on time scales consistent with the solar system and observations of protoplanetary disks. These scenarios are variants on models where continued agglomeration of small rocky solids yields a stable planetary system (e.g., Safronov 1969; Wetherill 1980). Solids not incorporated into planets provide material for excess emission from dust.

In the classical picture developed to explain the Solar System (e.g., Safronov 1969 ; Lewis 1972; Weidenschilling 1974; Wetherill 1980), the process starts with a disk of small solids having just enough mass (the Minimum Mass Solar Nebula, hereafter MMSN) to reproduce objects in the Solar System. Collisional processes merge small solids into kmsized or larger planetesimals, then Mars-mass protoplanets, and finally Earth mass planets. During the 'giant impact' phase when Mars-mass protoplanets merge into Earths, the surface density of the gaseous disk is probably $\lesssim 1 \%$ of the initial surface density; otherwise, gas drag circularizes the orbits of Mars-mass objects and prevents giant impacts (e.g., Kominami \& Ida 2002). Throughout the accumulation and "clean up" phases, high velocity collisions of leftover planetesimals, impacts of intermediate-sized protoplanets, and giant impacts of massive protoplanets convert $15 \%$ to $30 \%$ of the initial mass in solids into debris (Agnor et al. 1999; Kenyon \& Bromley 2004; Agnor \& Asphaug 2004; Goldreich et al. 2004; Raymond et al. 2011; Genda et al. 2015b).

In the pebble accretion scenario, dynamical processes within the gaseous disk concentrate cm-sized pebbles into large planetesimals with radii of 100-1000 km (e.g., Youdin \& Goodman 2005; Johansen et al. 2007; Youdin 2010; Johansen et al. 2015; Simon et al. 2016). Continued accretion of pebbles and mergers of planetesimals eventually produce a set of stable planets (e.g., Johansen et al. 2015; Levison et al. 2015; Chambers 2016). Current investigations of pebble accretion ignore the loss of material and debris production during mergers of large planetesimals. If fragmentation removes $5 \%$ to $10 \%$ of the initial mass (e.g., Johansen et al. 2015), neglecting this process has a limited impact on the formation of large planets. However, the mass lost through fragmentation is much larger than the sub-lunar mass of solids required to produce a detectable $24 \mu \mathrm{m}$ excess $(\S 2)$.

In the in situ planet formation scenario, Hansen \& Murray (2012) suggest that high concentrations of solids inside $1 \mathrm{AU}$ are required to explain various properties of the Kepler planet population ${ }^{1}$ (see also Kuchner 2004; Chiang \& Laughlin 2013; Hansen \& Murray 2013; Hansen 2015). Starting from an ensemble of protoplanets with a solid surface density $\sim 10$ times larger than the MMSN, a series of giant impacts produces a stable system of several

${ }^{1}$ Volk \& Gladman 2015) address aspects of this picture in the context of the solar system. 
planets in a few Myr (Hansen \& Murray 2013). Because the calculations start with assumed ensembles of fully formed protoplanets, Hansen \& Murray (2013) ignore the inefficiency of assembling solids into protoplanets and the debris produced by high velocity collisions of leftover planetesimals. As in recent explorations of pebble accretion, the numerical simulations also ignore the loss of material (and consequent debris production) in giant impacts.

For any of these scenarios, tidal torques can drive the radial migration of protoplanets through the gaseous disk (e.g., Ward 1997; Ida et al. 2000; Masset \& Papaloizou 2003; O’Brien et al. 2006; Papaloizou et al. 2007; Ida \& Lin 2008; Bromley \& Kenyon 2011; Raymond \& Cossou 2014). Planets may then form at large $a$ and migrate to small $a$. Although typical migration models do not consider collisional disruption of small solids inside 2 AU, largescale destruction seems likely. When Earth-mass and super-Earth-mass planets migrate inside 2 AU, they excite pre-existing smaller solids onto high $e$ orbits (e.g., Armitage 2003 ; Bromley \& Kenyon 2011; Walsh et al. 2011; Kenyon \& Bromley 2014). Destructive collisions among these objects then produce copious amounts of dust and a detectable $24 \mu \mathrm{m}$ excess (e.g., Wyatt 2008; Jackson \& Wyatt 2012).

\subsection{Modes of Dust Production}

To develop predictions for the magnitude of the IR excess produced during rocky planet formation, we identify likely epochs of dust production. In all of the scenarios discussed above, the growth of pebbles and larger planetesimals with $r \approx 1 \mathrm{~m}$ to $1000 \mathrm{~km}$ takes place in an opaque gaseous environment where debris mixes with pre-existing small particles (see Dauphas \& Chaussidon 2011, and references therein). Because we cannot distinguish 'primordial' dust from debris, we ignore this phase of dust production.

As solids grow from planetesimals into protoplanets and then planets, there are three modes of dust formation. Mergers of planetesimals into protoplanets typically produce modest amounts of debris (e.g., Wetherill \& Stewart 1993; Kenyon \& Luu 1999; Weidenschilling et al. 1997). As protoplanets grow, they stir the orbits of leftover planetesimals which never become incorporated into a planet. Among the planetesimals, high velocity collisions then begin to produce numerous smaller particles with sizes ranging from $1 \mu \mathrm{m}$ to tens of $\mathrm{km}$ Greenberg et al. 1978; Wetherill \& Stewart 1993; Kenyon \& Bromley 2004; Weidenschilling 2010; Raymond et al. 2011). This debris fuels a collisional cascade, where solids are ground down into sub-micron-sized particles which are ejected by radiation pressure. Eventually, giant impacts produce larger and larger protoplanets. Debris from giant impacts adds to the debris from collisions of leftover planetesimals and fuels the collisional cascade Jackson \& Wyatt 2012; Genda et al. 2015b). 
In all three scenarios, the growth of protoplanets, collisions of leftover planetesimals, and giant impacts generate debris. For simplicity, we ignore the modest amount of debris generated from the growth of protoplanets and derive separate estimates for dust produced from destructive collisions of leftover planetesimals $(\$ 4.2)$ and from giant impacts $(\$ 4.3)$.

\subsection{Debris from Collisions of Leftover Planetesimals}

In classical planet formation theory, semi-analytical and numerical calculations of collisions among ensembles of rocky planetesimals within 2-3 AU report debris production ranging from roughly $5 \%$ to almost $50 \%$ of the initial mass in solid material (Greenberg et al. 1978; Wetherill \& Stewart 1993; Kenyon \& Bromley 2004; Leinhardt \& Richardson 2005; Kenyon \& Bromley 2005; Chambers 2008; Weidenschilling 2010; Kenyon \& Bromley 2016). The typical mass in the debris is $10 \%$ to $20 \%$ of the initial mass. Significant numbers of destructive collisions begin early, at $~ 0.01-0.1 \mathrm{Myr}$, and last until 10-100 Myr (see also Morbidelli et al. 2012; Raymond et al. 2014; Quintana et al. 2016, and references therein).

In these calculations, the timing of debris production overlaps epochs when we expect a significant decay in the surface density $\Sigma_{g}$ of the gaseous disk (Hartmann et al. 1998; Haisch et al. 2001; Mamajek 2009; Williams \& Cieza 2011; Alexander et al. 2014). When $\Sigma_{g}$ is large, gas drag forces debris to spiral into the central star. As $\Sigma_{g}$ declines, the system retains a larger and larger fraction of the debris. For young stars with no gaseous disk at ages of 10-20 Myr, we conservatively estimate that $5 \%$ to $10 \%$ of the initial mass in solids is converted into debris. The formation of an Earth-mass planet thus generates 0.05-0.10 $\mathrm{M}_{\oplus}$ in debris, which is 10-20 times larger than the mass required to produce a detectable $24 \mu \mathrm{m}$ excess.

\subsection{Debris from Giant Impacts}

To predict debris production from giant impacts, we first consider a simple analytical model. In this approach, we compare the collision energy to the binding energy of a pair of protoplanets (see also Davis et al. 1985; Housen \& Holsapple 1990; Davis \& Ryan 1990; Housen \& Holsapple 1999). We assume conservatively that all collisions are head-on (impact parameter $b=0$ ); more glancing collisions typically yield more debris (e.g., Leinhardt \& Stewart 2012). For two protoplanets with mass $m$ and radius $r$, the center-of-mass collision energy is $Q_{c}=v_{i m p}^{2} / 8$ (e.g., Wetherill \& Stewart 1993; Kenyon et al. 2014; Kenyon \& Bromley 
2016), where the impact velocity $\left.\right|^{2}$ is

$$
v_{i m p}^{2} \approx v_{r e l}^{2}+v_{r e l}^{2}+v_{e s c}^{2} .
$$

Here, $v_{r e l}$ is the velocity of each protoplanet relative to a circular orbit and $v_{e s c}$ is the mutual escape velocity of the pair at the moment of the collision, $v_{e s c}=\sqrt{2 G(m+m) /(r+r)}=$ $\sqrt{2 G m / r}$. The fraction of material ejected during a collision is

$$
f_{e j}=0.5 \frac{Q_{c}}{Q_{D}^{\star}},
$$

where $Q_{D}^{\star}$ is the binding energy (e.g., Agnor et al. 1999; Benz \& Asphaug 1999; Canup \& Asphaug 2001; Leinhardt \& Richardson 2005; Leinhardt \& Stewart 2009; Genda et al. 2012, 2015a). When $Q_{c} \approx Q_{D}^{\star}$, half the mass of the merged pair is ejected to infinity.

Protoplanets typically have relative velocities of $25 \%$ to $75 \%$ of the escape velocity of the largest protoplanets. We set $v_{r e l} \approx e v_{K}$, where $v_{K}$ is the velocity of a circular orbit and $e$ is the eccentricity. When protoplanets have masses ranging from a lunar mass to an Earth-mass, $e \approx 0.1-0.2$ (e.g., Chambers 2001; Raymond et al. 2004, 2005; Kenyon \& Bromley 2006; Kokubo et al. 2006; Kokubo \& Genda 2010; Chambers 2013; Quintana et al. 2016).

For parameters appropriate for rocky objects at 1 AU (see $\$$ A.1), collisions between equal-mass protoplanets with $e \approx 0.05-0.2$ eject roughly $10 \%$ of the total mass (Fig. 2). Setting the relative velocity equal to $50 \%$ of the escape velocity of the protoplanet yields similar results. Although the accretion history of an Earth-mass object is complicated (e.g., Kenyon \& Bromley 2006; Kokubo et al. 2006; Chambers 2013), final assembly requires several collisions of sub-Earth mass objects. If each collision loses roughly $10 \%$ of the initial mass, the total amount of lost mass exceeds $0.1 \mathrm{M}_{\oplus}$. This mass is roughly an order of magnitude larger than the mass required to produce a detectable debris disk (\$2).

Detailed $n$-body and SPH calculations support this simple estimate (e.g., Agnor \& Asphaug 2004; Asphaug et al. 2006; Raymond et al. 2011; Genda et al. 2012; Stewart \& Leinhardt 2012; Jackson \& Wyatt 2012; Chambers 2013; Genda et al. 2015b). In SPH simulations, dust production depends on $b$ and $v_{r e l}$. Head-on collisions with small $b$ and $v_{r e l}$, yield little or no dust. When $v_{r e l}$ is large or the collision is oblique $(b \gtrsim 0.3-0.4)$, dust production is substantial. Averaged over a complete $n$-body simulation of an ensemble of growing protoplanets, protoplanet collisions disperse $10 \%$ to $20 \%$ of the initial mass into

\footnotetext{
${ }^{2}$ In our approach, both protoplanets have the same velocity relative to a circular orbit. Thus, the impact velocity includes a contribution from each one.
} 
small fragments. Thus, giant impacts involved in the formation of a single Earth-mass planet yield at least $0.1-0.2 \mathrm{M}_{\oplus}$ in debris.

\subsection{Evolution of IR Excess from the Debris of Planet Formation}

To derive predicted detection rates for warm debris disks, we adopt an initial disk mass $M_{0}$ and a model for the time evolution of the total mass $M_{d}$ and cross-sectional area $A_{d}$ of the debris produced through collisions of leftover planetesimals and giant impacts. In a real debris disk, stochastic collisions add and remove debris; thus, $M_{d}$ and $A_{d}$ decrease over the long term and increase and decrease on short timescales (e.g., Grogan et al. 2001; Kenyon \begin{tabular}{|l|l|l|l|l|}
\hline \& Bromley 2004; Weidenschilling 2010; Jackson \& Wyatt 2012; Genda et al. 2015b; Kenyon \\
\hline
\end{tabular} \& Bromley 2016). Instead of following this evolution in detail, we consider an analytical model where $A_{d}$ and $M_{d}$ decline monotonically with time (see $\$ \mathrm{~A} .1$. With this conservative assumption, we derive a lower limit for the expected IR excess from the debris at any time $t$. If the analytical model predicts much larger IR excesses than observed, then more extensive numerical calculations of planet formation will also yield much larger excesses than observed.

As outlined in the Appendix (\$A.1), the long-term evolution of the debris in the analytical model depends on the surface density of solids, the size of the largest object, and the orbital eccentricity of debris particles. The model assumes that collisions among objects with radii smaller than $r_{\text {max }}$ are completely destructive, producing debris with particle sizes smaller than $r_{\max }$. The cascade of collisions maintains a power-law size distribution with $N(r) \propto r^{-3.5}$ from the smallest size $r_{\min }$ up to $r_{\max }$. Radiation pressure ejects smaller particles. Protoplanets with radii larger than $r_{\max }$ are ignored. With destructive collisions for all particles smaller than $r_{\max }$ and ejection of material with $r \lesssim r_{\text {min }}$, the mass in solids steadily declines with time.

To predict the time evolution of dust in the terrestrial zone, we consider a disk with initial solid surface density $\Sigma_{s}(a)=x_{m} \Sigma_{0}\left(a / a_{0}\right)^{-3 / 2}$, inner radius $a_{i n} \approx 0.1 \mathrm{AU}$, and outer radius $a_{\text {out }} \approx 1-2 \mathrm{AU}$. This inner radius lies between the value adopted in some numerical calculations (0.05 AU, e.g., Hansen \& Murray 2012, 2013) and the 0.25 AU inner boundary for the Burke et al. (2015) analysis of Kepler data. Our results are insensitive to the exact value of $a_{i n}$. Disks with scale factor $x_{m}=1$ and $\Sigma_{0}=10 \mathrm{~g} \mathrm{~cm}^{-2}$ at $a_{0}=1$ AU have the surface density of the MMSN. For typical conditions in a disk with several protoplanets, $r_{\text {max }} \approx 100-1000 \mathrm{~km}$ and $e \approx 0.1$ Chambers 2008; Raymond et al. 2011; Kenyon \& Bromley 2016). If $r_{\max }$ and $e$ remain fixed throughout the evolution, the surface density declines roughly linearly with time, $\Sigma_{s}(t) \propto\left(1+t / t_{c}\right)^{-1}$, where $t_{c} \propto r_{\max } P / \Sigma_{0}$ is the collision time and $P$ is the orbital period. The relative luminosity of the debris disk is then an analytic 
function of the extent of the disk, the initial mass, and time (\$A.1).

At the start of the evolution, $L_{d} / L_{\star}$ depends only on $x_{m}$ and the radial extent of the disk (Appendix, eqs. A14-A15). The initial dust luminosity scales linearly with $x_{m}$ and with radial distance as $a^{-3 / 2}$. When $a_{\text {in }}=0.1 \mathrm{AU}$ and $a_{\text {out }}=2 \mathrm{AU}$, systems with $x_{m} \gtrsim 2 \times 10^{-4}$ have dust luminosity larger than the nominal detection limit, $L_{d} / L_{\star} \gtrsim 10^{-4}$.

Once the evolution begins, the dust luminosity declines on the local collision time. For a disk with $\Sigma_{s} \propto a^{-3 / 2}, t_{c} \propto a^{3}$. Although most of the mass is in the outer $\operatorname{disk}\left(M_{d} \propto a_{\text {out }}^{1 / 2}\right)$, the material closest to the star has the largest brightness per unit surface area. Solids close to the star also have the shortest collision time. Thus, the dust luminosity begins to decline on the collision time of the inner disk, $t_{i n}$. The luminosity declines by a factor of roughly two on this time scale (see also Kennedy \& Wyatt 2010).

On time scales larger than $t_{i n}$, collisions remove material from larger and larger disk radii. At the outer edge of the disk, the collision time is $t_{\text {out }}$. On time scales between $t_{\text {in }}$ and $t_{\text {out }}$, the dust luminosity declines rather slowly with time, $L_{d} / L_{\star} \propto t^{-n}$ with $n \approx 0.3-0.9$. Once the evolution time exceeds $t_{\text {out }}$, the decline in the dust luminosity follows the decline of a narrow ring, $L_{d} / L_{\star} \propto t^{-n}$ with $n=1$.

At late times, the dust luminosity of disks with different $x_{m}$ converges (e.g., Wyatt \& Dent 2002; Dominik \& Decin 2003). Although the initial disk luminosity scales with $x_{m}$, disks with large $x_{m}$ evolve more rapidly than disks with small $x_{m}$. This convergent luminosity depends on $r_{\max }, x_{m}$, and the extent of the disk.

The lower panel of Fig. 3 illustrates the evolution of $L_{d} / L_{\star}$ at $0.1-50 \mathrm{Myr}$ for $0.1-1 \mathrm{AU}$ debris disks with $r_{\max }=300 \mathrm{~km}, e=0.1$, and a range of $x_{m}$. Disks with $x_{m}=0.3(30 \%$ of the MMSN) evolve from $L_{d} / L_{\star} \approx 10^{-1}$ at $10^{3}$ yr to $L_{d} / L_{\star} \approx 10^{-2}$ at $0.1 \mathrm{Myr}$ to $L_{d} / L_{\star} \approx 10^{-3}$ at 3-4 Myr. These disks remain much brighter than the nominal detection limit until stellar ages of 30-40 Myr. At early times ( $t \lesssim 0.1 \mathrm{Myr}$ ), the dust luminosity roughly scales with $x_{m}$; a disk with $x_{m}=0.003$ has a dust luminosity 100 times smaller than a disk with $x_{m}=0.3$. Because disks with smaller $x_{m}$ have longer collision times, they evolve more slowly. It takes a disk with $x_{m}=0.003$ roughly $15-20$ Myr to decline from $L_{d} / L_{\star} \approx 10^{-3}$ to $L_{d} / L_{\star} \approx 10^{-4}$.

The upper panel of Fig. 3 shows that larger disks are always brighter (eq. A15 of the Appendix). With a factor of 8 larger collision time at the outer edge, a disk extending to 2 AU declines more slowly than a disk extending to 1 AU. For disks with $x_{m}=0.3$, the larger disk reaches the detection limit at $100 \mathrm{Myr}$ instead of 30-40 Myr. As $x_{m}$ decreases, however, the differences in evolution times become smaller. When $x_{m}=0.003$, the $0.1-2 \mathrm{AU}$ disk reaches the detection threshold at $25 \mathrm{Myr}$ instead of 15-20 Myr for the 0.1-1 AU disk. 
To derive predicted flux ratios for debris disks, we assume the solids radiate as blackbodies in equilibrium with radiation from the central star. For simplicity, $L_{\star}=1 L_{\odot} R_{\star}=$ $1 R_{\odot}$, and $T_{\star}=5780 \mathrm{~K}$ at all times. The disk temperature is then $T_{d}=280\left(a / a_{0}\right)^{-1 / 2} \mathrm{~K}$. With no analytic solution for the flux ratio, we divide the disk into a series of annuli, assign a temperature to each annulus, derive the time evolution of the cross-sectional area and emitted flux in each annulus, and add up the fluxes. In the Appendix, we show that numerical integrations of the dust luminosity agree well with analytic results.

Fig. 4 summarizes results for $0.1-2$ AU disks with $r_{\min }=1 \mu \mathrm{m}, r_{\max }=300 \mathrm{~km}$ and various $x_{m}$. At current sensitivity limits, robust 8-12 $\mu \mathrm{m}$ detections of warm dust at $10 \mathrm{Myr}$ require massive debris disks with $x_{m} \gtrsim 0.3$; detections at $8 \mu \mathrm{m}$ are strongly favored over those at $12 \mu \mathrm{m}$. Warm dust is much easier to detect at 16-24 $\mu \mathrm{m}$. Spitzer $16 \mu \mathrm{m}$ measurements of $\sim 10$ Myr old stars can detect debris disks with $x_{m} \gtrsim 0.03$. The sensitivity at $24 \mu \mathrm{m}$ is fairly remarkable: debris disks with $x_{m}$ as low as $1 \%$ of the MMSN are detectable.

We can use these results to interpret statistics for warm debris from \$2. Among stars younger than 10 Myr in the FEPS sample, 3\%, 7\%, and 20\% of the non-primordial disks have a detected $24 \mu \mathrm{m}$ excess above $50 \%, 30 \%$, and $10 \%$ of the stellar photosphere. From Fig. 4, these results allow us to conclude that $<3 \%$ of FEPS sources younger than 10 Myr have a warm excess consistent with $x_{m} \gtrsim 0.1$. The $3 \%$ upper limit arises because cold dust beyond 2 AU could produce some of the observed $24 \mu \mathrm{m}$ excess. Similarly, fewer than $7 \%$ of sources younger than $10 \mathrm{Myr}$ have a warm excess consistent with $x_{m} \gtrsim 0.03$.

The FEPS $16 \mu \mathrm{m}$ excess statistics place a more stringent limit on $x_{m}$. Because no FEPS source at any age (between $3 \mathrm{Myr}$ and $3 \mathrm{Gyr}$ ) has a $16 \mu \mathrm{m}$ excess above $16 \%$ of the stellar photosphere, all sources younger than 10 Myr must have $x_{m} \lesssim 0.05$. Similarly, all sources younger than 5 Myr have $x_{m} \lesssim 0.03$.

For a given $x_{m}$, reducing $r_{\max }$ reduces the predicted level of IR excess. Fig. 5 shows how the $24 \mu \mathrm{m}$ excess varies with $r_{\max }$ for 0.1-2 AU debris disks with $x_{m}=0.1$ and $r_{\min }=$ $1 \mu \mathrm{m}$. In systems with identical initial masses, swarms of particles with smaller $r_{\text {max }}$ have larger initial $A_{d}$ and smaller collision times. Although swarms with $r_{\max }=3-10 \mathrm{~km}$ initially have larger $24 \mu \mathrm{m}$ excesses than swarms with $r_{\text {max }}=1000 \mathrm{~km}$, they also evolve much more rapidly. By 6 Myr $(12 \mathrm{Myr})$, debris disks with $r_{\max }=3 \mathrm{~km}(10 \mathrm{~km})$ reach the nominal detection limit. Disks with $r_{\max }=100-1000 \mathrm{~km}$ take $40-100$ Myr to reach this limit. 


\subsection{Summary}

Numerical calculations of planet formation suggest that the agglomeration of Earthmass planets in the terrestrial zone creates copious amounts of debris. Current estimates suggest from $15 \%$ to $30 \%$ of the initial mass is potentially available to produce the debris signature of rocky planet formation. Debris production is probably independent of the mode - classical, in situ, or pebble - in which planets form. Unless planetesimal formation mechanisms assemble Mars-mass oligarchs directly, leaving behind $10 \%$ of the initial mass in debris is inevitable (e.g., Kenyon \& Bromley 2016). By generating smaller planetesimals, current formation paths appear to preclude this possibility (Johansen et al. 2015; Simon et al. 2016). All popular models for the formation of Earth-mass planets include a giant impact phase which also generates significant amounts of debris.

The debris is long-lived. In standard models of planet formation, the debris has a typical maximum size of 100-1000 km (e.g., Kenyon \& Bromley 2016). If the debris contains $15 \%$ to $30 \%$ of the initial mass required to assemble an Earth-mass planet, then the IR excess from the debris is detectable at $24 \mu \mathrm{m}$ for $\lesssim 100$ Myr (Fig. 4, see also Raymond et al. 2011; Genda et al. 2015b).

For stars with ages $\lesssim 10 \mathrm{Myr}$, it is challenging to detect debris from terrestrial planet formation with current sensitivity limits at 8-12 $\mu \mathrm{m}$. For robust detections, recent surveys require an initial solid mass $\gtrsim 30 \%$ of the MMSN at $8 \mu \mathrm{m}$ and $\gtrsim 100 \%$ of the MMSN at $12 \mu \mathrm{m}$. If the formation of several Earth-mass planets converts $15 \%$ to $30 \%$ of the initial mass into debris, detection at $12 \mu \mathrm{m}$ is very unlikely: the debris simply is not luminous enough after 10 Myr. At $8 \mu \mathrm{m}$, the predicted level of debris from the formation of a single Earth-mass planet is somewhat smaller than the Spitzer limits. If Earth-mass planets are as common as suggested by Kepler (Burke et al. 2015), the low frequency, $\lesssim 3 \%$ (e.g., Luhman \& Mamajek 2012), of warm dust revealed by $8 \mu \mathrm{m}$ observations of solar-type stars in the Upper Sco association is roughly consistent with theoretical predictions. However, if we

include the excesses produced by the formation of more massive planets $\left(\gtrsim 1 \mathrm{M}_{\oplus}\right)$, the expected $8 \mu \mathrm{m}$ excess is larger than Spitzer sensitivity limits and potentially in conflict with observations.

If our nominal picture of debris production is correct (Fig. 4), current 16-24 $\mu \mathrm{m}$ sensitivity limits are low enough to detect every solar-type star $\lesssim 10$ Myr engaged in forming Earth-mass planets. For 10 Myr old stars, current $16 \mu \mathrm{m}$ data should detect debris disks with initial masses of $5 \%$ to $10 \%$ of the MMSN; however, the FEPS survey detected none of these systems. Similarly, $<3 \%$ of FEPS sources younger than 10 Myr have a warm excess consistent with initial solid masses $\gtrsim 10 \%$ of the MMSN. 
Based on this analysis, there is a clear discrepancy between the observed frequency of warm debris from terrestrial planet formation $(\lesssim 3 \%$, §2) and the incidence rate of Earthmass planets derived from Kepler $(\gtrsim 20 \%$, §3). Based on Fig. 4, the observed frequency of 16-24 $\mu \mathrm{m}$ excess sources and the inferred incidence rate of Earth-mass planets imply that terrestrial planet formation must leave behind a very small fraction of the initial mass in debris-producing solids $(\lesssim 1 \%$ of $\mathrm{MMSN})$.

\section{DISCUSSION}

The low rate of warm excess among solar-type stars of terrestrial planet-forming age was previously noted in the early analysis of FEPS Spitzer/IRAC results. Because terrestrial planet formation should produce a detectable warm excess (Kenyon \& Bromley 2004), Silverstone et al. (2006) interpreted their non-detection of warm excesses as possible evidence that (i) terrestrial planets form infrequently or (ii) warm dust dissipates more quickly than expected (see also Carpenter et al. 2009b). At that time, the rarity of warm excesses was not particularly remarkable because the incidence rate of terrestrial planets was completely unknown. Our more recent understanding that Earth-mass planets may be fairly common now highlights the need to understand why warm excesses are rare.

In our analysis, the inferred discrepancy between the fraction $f_{d}$ of young solar-type stars with warm debris and the fraction $f_{p}$ of mature solar-type stars with Earth-mass planets $\left(f_{d} \lesssim 0.1 f_{p}\right)$ relies on our understanding of (i) recent Kepler planet detection statistics, (ii) updated statistics for 8-24 $\mu \mathrm{m}$ emission from disks around young stars, and (iii) new developments in planet formation theory. In this section, we consider each of these elements in turn to identify possible ways to resolve the discrepancy between $f_{d}$ and $f_{p}$. We then consider physical mechanisms that might reconcile the two frequencies and suggest paths to test these ideas.

\subsection{Frequency of Earth-Mass Planets at 0.25-2 AU Around Solar-Type Stars}

When the frequency of false-positives $f_{f p}$ among Kepler Earth-mass planet candidates is large, we overestimate $f_{p}$. Current analyses infer $f_{f p} \approx 10 \%$ to $75 \%$ (e.g., Morton \& Johnson 2011; Santerne et al. 2012; Fressin et al. 2013; Sliski \& Kipping 2014; Désert et al. 2015; Colón et al. 2015; Santerne et al. 2016; Coughlin et al. 2016; Morton et al. 2016). Many studies focus on gas giant planet candidates; however, Désert et al. (2015) consider whether Spitzer observations confirm transits for Earth-mass candidates with orbital periods $\lesssim 100 \mathrm{~d}$. 
For individual systems, they derive $f_{f p} \approx 1 \%$ to $42 \%$; the complete sample suggests a typical $f_{f p} \approx 10 \%$. Curiously, additional observations confirm planets in several candidate systems with large individual $f_{f p}$. Although larger samples are required to understand the true $\mathrm{f}_{f p}$ for Earth-mass planet candidates, these data suggest $f_{p}$ is not overestimated by a factor of 10 (see also Coughlin et al. 2016; Morton et al. 2016).

If multi-planet systems occur commonly and with a large range in orbital inclination $\imath$, the true $f_{p}$ is smaller than our estimates. Although transit surveys of such systems may detect only one of two or more possible planets, detecting a transiting planet in the system is more likely because a planet can be detected from multiple directions. As a result, the fraction of stars with planets in the habitable zone $f_{p}$ can be reduced while maintaining the same average number of Kepler planets per star overall.

Although observations do not yet provide a robust estimate of the frequency of multiplanet systems (e.g., Tremaine \& Dong 2012; Fabrycky et al. 2014; Van Eylen \& Albrecht 2015; Winn \& Fabrycky 2015, and references therein), comparing results from radial velocity and transit surveys provides useful constraints (S. Tremaine, private communication). Transit surveys can detect multi-planet systems only when the range of mutual inclinations is $\lesssim$ a few degrees; however, radial velocity surveys are sensitive to systems with a much broader range of mutual inclinations. The frequency of multi-planet systems from recent Kepler catalogs (0.19-0.20; Fabrycky et al. 2014; Mullally et al. 2015; Coughlin et al. 2016) is 2/3 of the frequency of $\sim 0.3$ derived from radial velocity measurements (Limbach \& Turner 2015). The similarity between the two rates implies that Kepler does not significantly underestimate the fraction of multi-planet systems, i.e., mutual inclinations are small and planetary systems are fairly flat.

While this argument applies to mutual inclinations of planets of all masses, additional dynamical considerations can restrict the mutual inclinations of systems of Earth-mass planets. For known multi-planet systems, the typical range in $\imath$ is small, $\approx 1^{\circ}-5^{\circ}$ (Tremaine \& Dong 2012; Fabrycky et al. 2014). With $e \approx 2-4 \imath$ (Fabrycky et al. 2014), $e \approx 0.035-0.20$. If this range in $e$ is typical of systems with Earth-mass planets, theory provides a guide to estimate a possible error in $f_{p}$ at $a=0.25-1 \mathrm{AU}$ (see also Tremaine 2015). From numerical simulations, systems of several Earth-mass planets have $e \approx 0.01-0.05$ and $\imath \approx e / 2$ (e.g., Chambers \& Wetherill 1998; Bromley \& Kenyon 2006; Raymond et al. 2007; Morishima et al. 2008; Chambers 2013). These systems are dynamically unstable when the distance between the apocenter of the inner orbit and the pericenter of the outer orbit exceed 10 mutual Hill radii $R_{H}$, where $R_{H}=\left(a_{\text {in }}+a_{\text {out }}\right)\left[\left(m_{\text {in }}+m_{\text {out }}\right) / 3 M_{\star}\right]^{1 / 3}$ and $a_{\text {in }}\left(a_{\text {out }}\right)$ is the semimajor axis of the inner (outer) planet (Chambers et al. 1996; Yoshinaga et al. 1999; Fang \& Margot 2013; $\mathrm{Pu} \& \mathrm{Wu} 2015 ;$ Petrovich 2015). When all planets have $e=0.03(e$ 
$=0.10$ ), the maximum number of stable Earth-mass planets within $0.4-1$ AU is $7-8(4-5)$. The maximum reduction in $f_{p}$ is then a factor of $5-7$.

The number of transiting planets in a multi-planet system depends on the distribution of mutual inclinations (e.g., Tremaine \& Dong 2012; Fabrycky et al. 2014). To make estimates for ensembles of closely packed Earth-mass planets, we perform a simple Monte Carlo calculation. For adopted dispersions in $e$ and $\imath, \sigma_{e}$ and $\sigma_{\imath}=\sigma_{e} / 2$, our algorithm establishes a set of Earth-mass planets with orbital separations of 10 mutual Hill radii and semimajor axes between $0.25 \mathrm{AU}$ and $1 \mathrm{AU}$. After randomly selecting one of these planets to have impact parameter $b \leq 1$, the code chooses random deviates for $\imath$, infers impact parameters for the remaining planets, and counts the number of planets with $|b| \leq 1$. For Gaussian (Rayleigh) deviates with $\sigma_{e} \approx 0.035$ and $\sigma_{\imath} \approx 1^{\circ}$, closely packed systems have seven (six) planets and four (three) transits per system. When $\sigma_{e} \approx 0.175$ and $\sigma_{\imath} \approx 5^{\circ}$, tightly packed systems have 3-4 (2-3) planets and one (one) transit per system. Kepler should detect maximally packed Earth-mass planets on roughly circular orbits as multi-planet systems, but should fail to identify multiple planets in highly eccentric systems. Either way, these results suggest $f_{p}$ might be overestimated by factors of $2-4$. Factor of ten overestimates are unlikely.

Overall, this analysis suggests that overestimates in $f_{p}$ are unlikely to reduce the fraction of stars with planets in the $0.25-1$ AU region $(\sim 20 \%)$ to the fraction of stars with warm excesses $(<3 \%)$. Moreover, our estimates are conservative. If there is a population of Earthmass planets with $a \approx 1-2 \mathrm{AU}$, then $f_{p}$ is larger than $20 \%$. If the typical $\sigma_{e}$ of multi-planet systems is roughly $4 \sigma_{\imath}$ instead of our adopted $2 \sigma_{\imath}$ (Fabrycky et al. 2014$)$, then the maximum number of Earth-mass planets in Kepler systems with a single transit is roughly two. Thus, uncertainties in $f_{p}$ cannot reduce the discrepancy between $f_{p}$ and $f_{d}$.

\subsection{Emission from Small Dust Grains}

Alternatively, we may have overestimated the expected IR excess produced by debris. To derive this excess $(\$ 4)$, we assume grains with sizes $\gtrsim 1 \mu \mathrm{m}$. In any debris disk model, the dust luminosity is sensitive to the size of the smallest grains, $L_{d} \propto r_{\min }^{-1 / 2}$. Grains with sizes smaller (larger) than our nominal limit of $1 \mu \mathrm{m}$ are more (less) luminous than predicted. Although $r_{\text {min }}$ is clearly a free parameter, observations of comets in the solar system and warm debris around solar-type stars suggest $1 \mu \mathrm{m}$ is a reasonable lower limit to the grain size (e.g., Lisse et al. 2006, 2007, 2008; Currie et al. 2011). Thus, increasing $r_{\min }$ seems an unlikely way to remove the discrepancy between $f_{d}$ and $f_{p}$.

We also assume disks with power-law size distributions of small particles. In a real 
collisional cascade, the equilibrium size distribution has pronounced waves relative to our adopted $N(r) \propto r^{-3.5}$ power law Campo Bagatin et al. 1994; O’Brien \& Greenberg 2003; Wyatt et al. 2011; Kenyon \& Bromley 2016). Adopting an analytic model for the waves which matches numerical simulations (Kenyon \& Bromley 2016) yields IR excesses a factor of 2-3 larger than the predictions of the basic analytical model outlined in the appendix. With this assumption, it is possible to detect debris disks with $10 \%$ to $30 \%(8-12 \mu \mathrm{m}), 1 \%$ $(16 \mu \mathrm{m})$, or $0.3 \%(24 \mu \mathrm{m})$ of the MMSN.

We also assume small grains radiate as perfect blackbodies. In a real debris disk, small (1-10 $\mu \mathrm{m})$ grains are probably hotter and radiate less efficiently than perfect blackbodies (e.g., Backman \& Paresce 1993; Dent et al. 2000; Lisse et al. 2006). If we adopt an extreme model where all grains radiate inefficiently (e.g., with emissivity $\epsilon \propto\left(\lambda / \lambda_{0}\right)^{-b}$, with $\lambda_{0}=$ $1 \mu \mathrm{m}$ and $\mathrm{b} \approx 0.8-1$ ), the IR excess at $8-24 \mu \mathrm{m}$ is roughly $50 \%$ smaller than the blackbody prediction. At 8-12 $\mu \mathrm{m}$, this prediction has little impact on our ability to detect excesses around 10 Myr stars: blackbody grains are already at or below the nominal detection limits for Spitzer and WISE. At 16-24 $\mu \mathrm{m}$, systems with initial masses of $10 \%(16 \mu \mathrm{m})$ or $1 \%$ $(24 \mu \mathrm{m})$ of the MMSN still remain above the detection threshold even with an extreme emission model.

Considering the uncertainties in $r_{\min }$ and the radiative properties and size distribution of small particles, our estimates for the IR excesses of warm debris disks seem reasonable. Allowing for changes in $L_{\star}$ from pre-main sequence stellar evolution (e.g., Baraffe et al. 1998; Siess et al. 2000; Bressan et al. 2012; Chen et al. 2014; Baraffe et al. 2015) and corresponding variations in dust temperature also has little impact on predicted IR excess emission at 8$24 \mu \mathrm{m}$. Thus, the discrepancy between $f_{d}$ and $f_{p}$ remains.

\subsection{Reconsidering Planet Formation Theory: Quick and Neat}

If we accept the currently measured frequencies of Earth-mass planets and warm debris disks, we must then devise a theory that assembles Earth-mass planets with little detectable debris.

One way to achieve this goal is if planet formation is quick. If Earth-mass planets orbiting solar-mass stars reach their final masses during the T Tauri phase, debris ejected from giant impacts or collisions of leftover planetesimals would be rapidly mixed with the optically thick primordial dust left within the gaseous disk. Instead of producing a distinctive 'debris disk signature,' the debris simply contributes to the already large IR excess of the primordial disk. As viscous evolution and other processes remove the gas, the 
debris from Earth-mass planet formation leaves with the gas.

In the classical theory, planet formation is not quick. Although protoplanet formation might occur during the T Tauri phase (e.g., Wetherill \& Stewart 1993; Weidenschilling 1997; \begin{tabular}{|l|l|l|l|}
\hline Ohtsuki et al. 2002; Kenyon \& Bromley 2006; Kokubo et al. 2006; Kokubo \& Genda 2010; \\
\hline
\end{tabular} Kenyon \& Bromley 2016), growth then stalls (e.g., Weidenschilling et al. 1997; Chambers 2001; Kenyon \& Bromley 2006; Raymond et al. 2007; Chambers 2008, 2013; Raymond et al. 2014; Genda et al. 2015b; Quintana et al. 2016). Once the gaseous disk dissipates, giant impacts among Mars-mass protoplanets then build Earth-mass planets (Kominami \& Ida 2004), generating debris. The gaseous disk plays no further role in the evolution of the debris.

Pebble accretion is not intrinsically quick (e.g., Chambers 2014; Johansen et al. 2015; Levison et al. 2015). Recent studies suggest the rapid formation of 100-1000 km planetesimals at $\lesssim 1$ Myr. These planetesimals rapidly accrete leftover pebbles. Because these models begin with initial masses comparable to the MMSN, the giant impact phase begins well after the gaseous disk has dissipated. Giant impacts then occur on time scales when debris is easily detected.

The massive disks of solids proposed in the in situ theory ( $\sim 10$ times the MMSN) enable rapid planet formation (Hansen \& Murray 2012, 2013; Hansen 2015). Formation times scale inversely with the mass of solids; thus, protoplanets grow much faster than in the classical or pebble accretion theories. Even in a primordial gaseous disk, the large number of closely packed protoplanets generates a series of giant impacts, leading to multiple Earthmass planets in a few Myr. The gas can then remove small particles produced in giant impacts. However, the gas probably cannot remove any leftover planetesimals or other 1$100 \mathrm{~km}$ particles generated during the giant impact phase. To produce a $24 \mu \mathrm{m}$ excess that is $<10 \%$ of the stellar photosphere in a system where the initial mass in solids is $\sim 10$ times the MMSN, the giant impact phase must leave behind less than $0.1-0.2 \%$ of the initial mass in 100-1000 km objects (including any leftover planetesimals). Otherwise, the in situ theory makes too much debris at 10 Myr.

Thus, another way to satisfy the observational constraints is if planet formation is intrinsically neat: Earth-mass planets assemble with negligible dust emission. As described above, the classical, in situ, and pebble accretion scenarios are not neat enough to match observational constraints. To isolate the appropriate physical conditions for a 'neat' scenario, we rely on the analytical model outlined in the appendix A.1) and published numerical simulations (e.g., Kenyon \& Bromley 2016). To match current sensitivity limits at $16 \mu \mathrm{m}$ and $24 \mu \mathrm{m}$, we must reduce dust production by at least a factor of ten (i.e., reducing $x_{m}$ from $15 \%-30 \%$ to $<3 \%$; Fig. 4 ). In all numerical simulations, dust 
production depends on $v_{i m p} / Q_{D}^{\star}$, where $v_{i m p}$ is the impact velocity and $Q_{D}^{\star}$ is the binding energy. Simple dynamics sets $v_{i m p}$; changing it significantly is unlikely.

The binding energy is based on analytical and numerical simulations using state-ofthe-art equations of state. While increasing $Q_{D}^{\star}$ seems unlikely, it is worth exploring the required physics. As a simple comparison, the gravitational binding energy per unit mass of a uniform sphere exceeds the $Q_{D}^{\star}$ for basalt only when $r \gtrsim 10 \mathrm{R}_{\oplus}$. Thus, the internal degrees of freedom of protoplanets are important in setting $Q_{D}^{\star}$. While it may be possible to modify these by factors of 2-3, order of magnitude changes seem unlikely (see also Housen \& Holsapple 1999; Holsapple et al. 2002, and references therein).

If dust production cannot be limited, the main alternative is to reduce the lifetime of the debris. In this approach, a shorter debris lifetime lessens the likelihood of detection. From eqs. A4 and A10, lowering $r_{\max }$ or increasing $v_{i m p} / Q_{D}^{\star}$ by a factor of ten shortens the lifetime of the debris by a similar factor. Having already ruled out factor-of-ten changes in $v_{\text {imp }} / Q_{D}^{\star}$, we consider the impact of changing $r_{\text {max }}$.

In the analytical model, changing $r_{\max }$ modifies the detectability of the debris in two ways. For a fixed mass in debris, the cross-sectional area $A_{d} \propto r_{\max }^{-1 / 2}$. Reducing $r_{\max }$ therefore increases the initial dust luminosity and shortens the collision time. As shown in Fig. 5, $r_{\max }$ must be quite small to reduce the predicted warm excess to the level of the $24 \mu \mathrm{m}$ excess displayed by the brightest $20 \%$ of $\sim 10 \mathrm{Myr}$ old stars. If $x_{m}=0.1$ and $r_{\max }=10 \mathrm{~km}$, then at $10 \mathrm{Myr} F_{d} / F_{\text {star }} \sim 0.12$ at $24 \mu \mathrm{m}$. Approximately $20 \%$ of FEPS sources younger than $10 \mathrm{Myr}$ have an excess brighter than $F_{d} / F_{\text {star }} \sim 0.1$ at $24 \mu \mathrm{m}$, a fraction comparable to the fraction of solar-type stars with Earth-like planets.

Although this solution seems attractive, it has several major drawbacks. Numerical simulations of giant impacts already yield $24 \mu \mathrm{m}$ fluxes much larger than observed (Genda et al. 2015b); generating a smaller likelihood of detection at $10 \mathrm{Myr}$ age in exchange for a much larger initial luminosity is probably a poor trade. For dust generated from planetesimals, numerical simulations suggest that collisional damping of small particles maintains large dust luminosities far longer than suggested by the analytical model (Kenyon \& Bromley 2016). Increasing the population of small particles by lowering $r_{\max }$ probably exacerbates this problem.

\subsection{Neat Planet Formation in a Remnant Gas Disk}

If we cannot substantially alter the observed frequency of terrestrial planets, models for the assembly of terrestrial planets, or the amount of debris generated by terrestrial 
planets, some process must remove small particles with $r \lesssim 0.1-1 \mathrm{~mm}$ from the terrestrial zone. Possibilities include interactions with the stellar radiation field, the stellar wind, or a remnant gaseous disk. If one of these processes removes small particles faster than the collisional cascade produces them, the predicted IR emission from warm dust is reduced by the requisite factor of $\sim 10$, eliminating the discrepancy between $f_{d}$ and $f_{p}$.

Compared to aerodynamic drag from a remnant gaseous disk, other mechanisms probably have a limited role. Observations suggest radiation pressure may remove particles smaller than $1 \mu \mathrm{m}$, but larger particles are robustly detected (e.g., Lisse et al. 2008; Currie et al. 2011; Matthews et al. 2014, and references therein). For $L_{d} / L_{\star} \gtrsim 10^{-7}$, Poynting-Robertson drag is ineffective (Wyatt 2008). In the inner Solar System, stellar wind drag is roughly $30 \%$ as effective as Poynting-Robertson drag in removing small particles (Gustafson 1994). Observations of the youngest solar-type stars suggest mass loss rates $\lesssim 10$ times the mass loss rate $\dot{M}_{\odot}$ of the current Sun (Wood et al. 2014, and references therein). Theoretical studies predict mass loss rates up to $100 \dot{M}_{\odot}$ (e.g., Cohen \& Drake 2014; Airapetian \& Usmanov 2016). Both of these estimates fall well below the $1000 \dot{M}_{\odot}$ required for stellar wind drag to remove small particles rapidly when $L_{d} / L_{\star} \gtrsim 10^{-4}$. Finally, the sputtering rate for small particles is too small compared to the collision rate (Wurz 2012).

In some circumstances, photophoresis ${ }^{3}$ drives small particles radially outward through an optically thin gaseous disk (e.g., Krauss \& Wurm 2005; Herrmann \& Krivov 2007; Cuello et al. 2016). When photophoresis is effective, the time scale for radial drift is comparable to (and sometimes shorter than) the time scale for radial drift due to gas drag. However, the drift time is sensitive to the ratio of the heat conductivity to the particle asymmetry factor, which is uncertain (e.g., von Borstel \& Blum 2012).

Here, we focus on the impact of gas drag, where the physical properties of small particles are less important. Our goal is to identify a range of $\Sigma_{g}$ for the gaseous disk that allows giant impacts $(0.1-1 \%$ or less of the MMSN) and removes $0.1-1 \mathrm{~mm}$ and smaller particles on time scales shorter than the collision time.

Within any gaseous disk, particles weakly bound to the gas rapidly drift inward when their 'stopping time' $\left(t_{s}=m v_{d} / F_{d}\right.$, where $v_{d}$ is the drift velocity and $F_{d}$ is the drag force) is comparable to their orbital period $P$ (Adachi et al. 1976a; Weidenschilling 1977a). At $1 \mathrm{AU}$, the shortest drift time is 50-100 yr (Fig. 6), shorter than the typical collision time of $1000 \mathrm{yr}$ for $1 \mu \mathrm{m}$ to $1 \mathrm{~cm}$ particles. In an optically thin disk, radiation pressure drives small,

\footnotetext{
${ }^{3}$ The photophoresis mechanism exploits the temperature gradient across an illuminated grain embedded in a low pressure gas. Momentum exchange with the gas results in the grain moving away from the radiation source.
} 
weakly coupled particles to large $a$ where the particles are colder and have much smaller dust luminosities (Takeuchi \& Artymowicz 2001). Effective radial drift thus eliminates particles that produce an IR exces: 4 .

To quantify radial drift at $1 \mathrm{AU}$ in a low density gaseous disk, we combine the approaches of Weidenschilling (1977a) and Takeuchi \& Artymowicz (2001). As outlined in the Appendix ( $\$$.2), we solve for the radial and azimuthal velocity of particles relative to the gas in a protostellar disk with standard relations for the surface density, midplane temperature, vertical scale height, gas pressure, thermal velocity, and other physical variables (e.g., Kenyon \& Hartmann 1987; Chiang \& Goldreich 1997; Rafikov 2004; Chiang \& Youdin 2010; Youdin \& Kenyon 2013; Armitage 2013).

Fig. 6illustrates the impact of a residual gas disk on the radial drift velocity of particles as a function of size at $1 \mathrm{AU}$ under MMSN-like conditions. For this example, a disk with the surface density of the MMSN has $\Sigma_{g}=2000 \mathrm{~g} \mathrm{~cm}^{-2}, T=278 \mathrm{~K}$, and vertical scale height $H=0.03$ AU. Filled circles indicate inward drift; open symbols indicate outward drift. Other choices for these parameters - e.g., $T=150-300 \mathrm{~K}$ and $H=0.02-0.10$ - change the maximum drift velocity and the particle size for this maximum drift by $25 \%$ to $50 \%$ but do not change the overall trends.

When the disk has $\Sigma_{g}$ comparable to the MMSN (Fig. 6, black symbols), particles with $r \approx 50 \mathrm{~cm}$ have $\tau_{s} \approx 1$ and the largest drift velocity. Inflection points in $v_{\text {rad }}(r)$ occur when particles enter different drag regimes (e.g., Epstein, Stokes, quadratic). For particle sizes $r \approx$ 30-40 $\mu \mathrm{m}$, outward radiation pressure balances inward gas drag; these particles do not drift. Smaller particles drift outward 5 with maximum velocities slightly smaller than $0.1 \mathrm{~cm} \mathrm{~s}^{-1}$

As the surface density of the gaseous disk declines, smaller particles drift more rapidly through the gas. Although the peak in $v_{\text {rad }}$ shifts to smaller sizes, the maximum drift velocity is always roughly $8000 \mathrm{~cm} \mathrm{~s}^{-1}$. The balance between radiation pressure and gas drag remains fixed for 30-40 $\mu \mathrm{m}$ particles. However, in disks with lower $\Sigma_{g}$, smaller particles have larger stopping times. With a weaker drag force, radiation pressure drives these particles outward at larger velocities. Once $\Sigma_{g}$ is roughly $0.001 \%$ of the MMSN, the outward drift velocities of $r \lesssim 30 \mu \mathrm{m}$ particles surpass the maximum inward drift velocities of $100 \mu \mathrm{m}$ particles.

\footnotetext{
${ }^{4}$ In a dense gaseous disk, interactions with the gas rapidly circularize the orbits of small particles (e.g., Adachi et al. 1976b Weidenschilling 1977a). During a collisional cascade in a low density disk, gravitational stirring by protoplanets raises $e$ faster than gas drag lowers $e$ (e.g, Kenyon \& Bromley 2004 Raymond et al. 2011, Genda et al. 2015b). Thus, we ignore this process.

${ }^{5}$ MMSN disks are probably optically thick. Small particles in the optically thin upper layers drift outward, while those in the optically thick midplane are unaffected.
} 
To judge the impact of radial drift on the collisional cascade, we compare the drift time to the collisional time. For disks with surface densities of $0.001 \%$ of the MMSN, $1-10 \mu \mathrm{m}$ and $100 \mu \mathrm{m}$ particles at 1 AU drift inward or outward on 50-75 yr time scales. In less (more) tenuous gas disks, particle lifetimes are longer (shorter; Fig. 6). For comparison, in a collisional cascade at $0.1-2 \mathrm{AU}$ with $L_{d} / L_{\star} \approx 10^{-4}$, the collision time is roughly $1000 \mathrm{yr}$. In disks with $\Sigma_{g} \approx 0.001 \%$ of the MMSN, radial drift from radiation pressure and gas drag removes particles smaller than $100 \mu \mathrm{m}$ in $<100 \mathrm{yr}$, rapidly depleting the disk of the $1-$ $100 \mu \mathrm{m}$ particles that comprise $90 \%$ of the surface area. Gas drag also rapidly transports larger $(0.1-1 \mathrm{~mm})$ particles towards the central star.

These two processes have a dramatic effect on debris production. In a standard collisional cascade, mass flows at a roughly constant rate from the largest particles to the smallest particles (e.g., Wyatt 2008; Kobayashi \& Tanaka 2010; Wyatt et al. 2011). When the gas drags $0.1-1 \mathrm{~mm}$ particles inward, the equilibrium population of these particles drops. Collisions among these objects are then less frequent, depressing the flow of mass to smaller particles. In turn, a smaller mass flow rate lowers the cross-sectional area of the swarm and weakens the infrared excess.

When gas drag and radiation pressure effectively remove small particles inside 2 AU, the IR excess drops dramatically. For disks with $0.001-0.01 \%$ of the MMSN, the dust luminosity drops by 2-4 orders of magnitude. If this remnant disk extends close to the stellar photosphere, particles with sizes $\gtrsim 1 \mathrm{~mm}$ either evaporate or fall onto the photosphere. Thus, residual gas disks as dilute as $0.001 \%$ of the MMSN can remove the small solids $(<100 \mu \mathrm{m})$ that make up $90 \%$ of the cross-sectional area of the debris, reducing the IR excess at $5-$ $20 \mathrm{Myr}$ to a level consistent with the observational constraints. Less dilute gas disks $(0.01 \%$ of the MMSN) can reduce the population of small grains $<100 \mu$ m indirectly, by removing the 1-10 mm solids that generate them through collisions. More dilute gas disks are less efficient in removing solids $>10 \mu \mathrm{m}$ and therefore less able to limit the IR excess.

We conclude that a residual gas disk in the range $0.001-1 \%$ of the MMSN that persists for $\sim 10 \mathrm{Myr}$ is dilute enough to allow giant impacts to assemble terrestrial planets in classical planet formation, but dense enough to minimize the observable IR excess produced by the resulting debris.

\subsection{Observational Constraints on Residual Gas Disks}

Current observational constraints on the surface density of residual circumstellar gas disks cannot exclude our picture for reducing IR excesses around 10 Myr old solar-type 
stars. Constraints on the residual gas content of disks from in situ diagnostics are modeldependent (Gorti \& Hollenbach 2004) and not highly restrictive in this context. For example, analyses of Spitzer IRS emission line diagnostics for a sample of FEPS targets with little evidence for warm debris disks place a formal limit on the gas surface density $\Sigma_{g}<0.01 \%$ of MMSN beyond $1 \mathrm{AU}$ (Pascucci et al. 2006). If this upper limit were to extend inside $1 \mathrm{AU}$, the surface density would be low enough to allow giant impacts in the classical planet formation picture (e.g., Kominami \& Ida 2002, 2004) and large enough to allow the removal of small particles by gas drag.

Measuring stellar accretion is an alternate way to search for evidence of a residual gas disk. While accretion indicates that residual gas is present, it does not directly measure $\Sigma_{g}$. To obtain a rough scaling between stellar accretion rate $\dot{M}_{\star}$ and $\Sigma_{g}$, we can consider the average accretion rates of classical $\mathrm{T}$ Tauri stars at $1-10 \mathrm{Myr}$ age. Stellar accretion rates decrease from $\sim 10^{-8} M_{\odot} \mathrm{yr}^{-1}$ at the age of Taurus-Auriga (few Myr) to $\sim 4 \times 10^{-10} M_{\odot} \mathrm{yr}^{-1}$ at 10 Myr (Sicilia-Aguilar et al. 2006), i.e., only a factor of 25. If the fractional decrement in $\dot{M}_{\star}$ indicates a similar reduction in $\Sigma_{g}$, an accretion rate of $\sim 4 \times 10^{-10} M_{\odot} \mathrm{yr}^{-1}$ corresponds to a surface density at $1 \mathrm{AU}$ of $4 \mathrm{~g} \mathrm{~cm}^{-2}$ if the initial state is an $\alpha$-disk with a surface density of $100 \mathrm{~g} \mathrm{~cm}^{-2}$ at $1 \mathrm{AU}$ (Hartmann et al. 1998) or $80 \mathrm{~g} \mathrm{~cm}^{-2}$ if the initial state is the MMSN with a surface density of $2000 \mathrm{~g} \mathrm{~cm}^{-2}$ at $1 \mathrm{AU}$ (Weidenschilling 1977b). Non-accreting sources, with presumably lower $\Sigma_{g}$, make up more than $90 \%$ of young stars at $5-10$ Myr age (Fedele et al. 2010).

Based on the $\dot{M}_{\star}-\Sigma$ scaling relation, it appears that we need to probe very low accretion rates $\sim 10^{-13}-10^{-12} M_{\odot} \mathrm{yr}^{-1}$ to reach $\Sigma_{g} \lesssim 10^{-5}-10^{-4}$ of the MMSN and to exclude the possibility that 5-10 Myr old disks contain enough residual gas to erase a debris disk signature. For approximately solar mass stars with ages of 5-10 Myr, chromospheric emission limits our ability to measure stellar accretion rates below $\sim 10^{-10} M_{\odot} \mathrm{yr}^{-1}$ using $U$-band excesses (Ingleby et al. 2011b) or hydrogen emission line fluxes (Manara et al. 2013). H $\alpha$ emission line widths (Natta et al. 2004) and line profile analyses (Muzerolle et al. 1998ba) can probe lower accretion rates (e.g., Muzerolle et al. 2000; Manara et al. 2013).

From the shape and velocity extent of the $\mathrm{H} \alpha$ line profile (White \& Basri 2003; Natta et al. 2004), Riviere-Marichalar et al. (2015) derive a broad range of accretion rates for 11 sources in the $8 \mathrm{Myr}$ old $\eta$ Cha group. Six sources have $\dot{M}_{\star} \gtrsim 10^{-10} M_{\odot} \mathrm{yr}^{-1}$ from at least one spectrum; the other five sources have $\dot{M}_{\star} \approx 10^{-12}-3 \times 10^{-11} M_{\odot} \mathrm{yr}^{-1}$. While it may not be appropriate to apply the Natta et al. (2004) relation derived for low mass stars to a set of solar-type stars, these accretion rates are close to the rates required for gas drag to eliminate the IR excesses of debris disks.

There are multiple prospects for detecting a dilute reservoir of gas in solar-type stars. 
CO fundamental emission (e.g., Najita et al. 2003; Salyk et al. 2011, Doppmann, Najita, \& Carr 2016) and UV transitions of molecular hydrogen (e.g., Ingleby et al. 2011a) probe molecular gas within an AU of young stars. Among A-type stars with debris disks, several have neutral or ionized gas close to the star (e.g., Hobbs et al. 1985; Ferlet et al. 1987; Welsh et al. 1998; Redfield 2007; Montgomery \& Welsh 2012; Kiefer et al. 2014; Cataldi et al. 2014, and references therein). Evaporation of comets (Lagrange et al. 1987; Beust et al. 1990) and vaporization of colliding particles (e.g., Czechowski \& Mann 2007) might supply some of this material (see also Kral et al. 2016).

\subsection{Testing the Possibilities}

In our analysis, we have considered four main options to resolve the discrepancy between the frequencies of warm debris disks $f_{d}$ and Earth-mass planets $f_{p}$ among solar-type stars. Despite clear gaps in our understanding of the physics of terrestrial planet formation, there is no obvious way to modify the theories to limit the detectability of warm debris at 5-20 Myr. Uncertainties in our ability to relate IR excesses to debris from rocky planet formation are unlikely to change $f_{d}$. Although overestimating the observed frequency of Earth-mass planets is a plausible cause for the difference between $f_{d}$ and $f_{p}$, the 'best' explanation is rapid drift of small particles in a residual gaseous circumstellar disk.

Future observations and theoretical investigations can test all four explanations. We outline several possibilities.

- Search for fainter warm excess signatures in large samples of young solar-type stars. Current sensitivity limits are not much lower than theoretical predictions. If warm excesses are simply a factor of 2-3 fainter than predicted, detecting these excesses with more sensitive surveys (e.g., using MIRI on JWST; Wright et al.|2003; Wells et al.|2006; Bouchet et al. 2015) may be able to distinguish neat scenarios from quick scenarios. For example, placing stricter limits on the level of $16 \mu \mathrm{m}$ excess $(\lesssim 10 \%$ of the stellar photosphere) would probe initial solid masses in debris of a few percent of MMSN (Fig. 4).

- In Kepler systems with apparent Earth-mass planets, better limits on (i) the falsepositive rate, (ii) the frequency of multiple planets, and (iii) the distributions of $e$ and $\imath$ in systems with multiple Earth-mass planets would reduce the uncertainties in the fraction of stars that host Earth-mass planets inside 1-2 AU. Theoretical studies into the stability of systems with multiple Earth-mass planets would place constraints on the ability of these systems to 'hide' from radial velocity and transit observations. 
- Hunt for planets around all young (5-20 Myr) solar-type stars. Several recent studies identify massive planets orbiting several pre-main sequence stars (e.g., Mann et al. 2016; Gaidos et al. 2016; David et al. 2016; Donati et al. 2016; Johns-Krull et al. $2016 \mathrm{~b}$ a). Identifying Earth-mass planets orbiting young stars would constrain the timescale of terrestrial planet formation relative to the production of debris. The K2 mission (Howell et al. 2014) might identify more short-period planets within several nearby young stellar associations ${ }^{6}$. TESS (Ricker et al. 2015; Sullivan et al. 2015) can search for planets with a much larger range of orbital periods.

- Search for evidence of tenuous residual gas disks, at the $10^{-5}-10^{-2}$ of MMSN level, around young solar-type stars. Direct detection of gas or robust measurement of very low mass accretion rates tests the idea that radiation pressure and aerodynamic drag remove the debris of terrestrial planet formation. In addition to surveys with the NIRSPEC or MIRI spectroscopic instruments on JWST (e.g., Wright et al. 2003; Wells et al. 2006), sensitive optical (e.g., Xu et al. 2016) or radio (e.g., ALMA, VLA) observations might reveal low mass gaseous disks in the terrestrial zones of 5-20 Myr old solar-type stars.

- Examine quick and neat modes of planet formation. Although our current understanding appears to preclude these ideas, it is important to quantify the ability of planet formation scenarios to assemble planets quickly and neatly in the absence of a long-lived gaseous disk. Future theoretical calculations of terrestrial planet formation should include clear predictions of dust production for comparison with observations (e.g., Raymond et al. 2011; Genda et al. 2015b; Kenyon \& Bromley 2016).

- Consider the late stages of protostellar disk evolution in more detail. Current mechanisms for disk dispersal (e.g., photoevaporation and viscous evolution) do not make firm predictions for the structure of gaseous material on time scales of 10-100 Myr (e.g., Gorti et al. 2015, and references therein). For example, including the gas from the evaporation of comets or vaporization of colliding planetesimals in photoevaporation models would help to constrain the long-term evolution of debris and gas in the terrestrial zones of young stars.

${ }^{6}$ Among the candidates reported in recent analyses of the K2 data (e.g., Foreman-Mackey et al. 2015. Vanderburg et al. 2016), 205117205.01 matches the position of an M2 pre-main sequence star in the Upper Sco association, 2MASS J16101473-1919095 (Luhman \& Mamajek 2012). IR data suggest the star has a debris or evolved transitional disk. However, the transit depth is very uncertain. Clarifying the existence and depth of transits in this system would begin to place constraints on the frequency of planets in the youngest stars. 


\section{SUMMARY}

In the past decade, detailed analyses of Kepler, Spitzer, and WISE data have established estimates for the frequencies of Earth-mass planets and warm dust in the terrestrial zones of solar-type stars. Although rocky planets are fairly common, the expected dusty 'signature' of terrestrial planet formation among 5-20 Myr old stars is a rare phenomenon. Potential explanations for the discrepancy include the possibility that (i) terrestrial planets are much less common than believed, (ii) planet formation is quicker and/or neater than predicted, and (iii) some physical mechanism removes warm dust rapidly. Although we cannot preclude some combination of the first two options, gas drag and radiation pressure can efficiently eliminate warm dust particles from the terrestrial zone when a residual gaseous circumstellar disk has a surface density of $>10^{-5}$ of the MMSN. Current constraints on the gas surface density within 1 AU of 5-10 Myr old solar-type stars are consistent with this limit. Thus, tenuous reservoirs of gas may impact our ability to observe the debris produced by rocky planet formation. If they do, warm debris is not a reliable signpost of rocky planet formation.

Modifying planet formation theory to resolve the discrepancy is tenable if planet formation is much more efficient than currently predicted by theory and leaves behind little debris. This scenario echoes the results of several previous studies (e.g., Greaves \& Rice 2010; Najita \& Kenyon 2014) that attempt to reconcile the inventory of solids bound up in known populations of exoplanets with the solid masses of protoplanetary disks. Producing the known exoplanet systems from the limited solid reservoirs in protoplanetary (Class I) disks requires a planet formation efficiency of roughly $30 \%$. If there is a substantial population of undiscovered planets or if planet formation is a messy process that discards solids by producing significant debris, the required efficiency of planet formation rises.

In the scenarios we investigate, typically $\sim 80 \%(20 \%)$ of the initial solid mass ends up in rocky planets (debris). To limit debris production, it is advantageous to start the planet formation process with an initial mass in solids reasonably close to the final mass in stable planets. Without some process that removes small grains from the disk, theoretical scenarios that invoke much larger initial mass reservoirs (e.g., Hansen \& Murray 2012, 2013; Hansen 2015; Volk \& Gladman 2015; Levison et al. 2015) should produce very large IR excesses which violate existing constraints from observations of 5-20 Myr solar-type stars.

In this sense, collisional debris can place strong constraints on planet formation scenar-

ios. As outlined in $\$ 45$, stringent tests require (i) tighter observational constraints on warm dust and residual disk gas during the expected epoch of terrestrial planet formation and (ii) planet assembly simulations which include the effect of gas drag in a residual gas disk. 
We acknowledge a generous allotment of computer time on the NASA 'discover' cluster. We thank G. Herczeg for valuable discussions of stellar accretion rates. Comments from and discussions with S. Andrews, J. Carpenter, M. Geller, A. Glassgold, G. Kennedy, N. Murray, I. Pascucci, D. Wilner, and an anonymous referee improved our presentation. Portions of this project were supported by the NASA Outer Planets Program through grant NNX11AM37G. The work of JN was performed in part at the Aspen Center for Physics, which is supported by National Science Foundation grant PHY-1066293. JN also acknowledges the stimulating research environment supported by NASA Agreement No. NXX15AD94G to the Earths in Other Solar Systems program.

\section{A. Long-term Evolution and Gas Drag in Debris Disks}

\section{A.1. Analytic Theory for an Extended Disk}

Wyatt \& Dent (2002) and Dominik \& Decin (2003) first developed an analytic theory to track the long-term evolution of collisional cascades (see also Wyatt et al. 2007a b; Heng \& Tremaine 2010; Kobayashi \& Tanaka 2010; Wyatt et al. 2011; Kenyon \& Bromley 2016). In this model, particles with sizes ranging from $r_{\min }$ to $r_{\max }$, orbital period $P$, and orbital eccentricity $e$ occupy a single annulus with width $\delta a$ at a distance $a$ from a central star with mass $M_{\star}$, radius $R_{\star}$, and luminosity $L_{\star}$. Particles collide at a rate $n \sigma v$, where $n$ is the number density, $\sigma$ is the physical cross-section, and $v$ is the collision velocity. Setting the center-of-mass collision energy $y^{7} Q_{c}$ larger than the binding energy $Q_{D}^{\star}$ of the largest particles ensures destructive collisions which produce debris. For an initial surface density of solids $\Sigma_{0}$ in the annulus, the collision time is

$$
t_{c}=\frac{\alpha r_{\max } \rho P}{12 \pi \Sigma_{0}}
$$

where the correction factor is $\alpha \approx \alpha_{0}\left(v^{2} / Q_{D}^{\star}\right)^{-n}$ (Wyatt et al. 2007a; Heng \& Tremaine 2010; Kobayashi \& Tanaka 2010; Wyatt et al. 2011). The surface density of material in the annulus then evolves as

$$
\Sigma_{s}(t)=\frac{\Sigma_{0}}{1+t / t_{c}}
$$

Other collective properties of the particles - including the total mass, cross-sectional area, and relative luminosity - follow the decline of $\Sigma_{s}$ with time (Wyatt \& Dent 2002; Dominik \& Decin 2003; Wyatt et al. 2007a; Kobayashi \& Tanaka 2010; Wyatt et al. 2011, KB2016).

\footnotetext{
${ }^{7}$ In our approach, the center-of-mass collision energy is $Q_{c}=\mu v^{2} / 2\left(m_{1}+m_{2}\right)$, where the reduced mass $\mu=m_{1} m_{2} /\left(m_{1}+m_{2}\right)$
} 
Here we expand the analytic theory to a disk extending from an inner radius $a_{i n}$ to an outer radius $a_{\text {out }}$. Particles with sizes ranging from $r_{\min }$ to $r_{\max }$ have an initial surface density

$$
\Sigma_{s}=x_{m} \Sigma_{0}\left(a / a_{0}\right)^{-p}
$$

where $\Sigma_{0}=10 \mathrm{~g} \mathrm{~cm}^{-2}$ is the surface density of solids at $a_{0}=1 \mathrm{AU}$ in the MMSN and $x_{m}$ is a scale factor (Weidenschilling 1977b; Hayashi 1981; Kenyon \& Bromley 2008). We assume that the correction factor $\alpha=\alpha_{0}\left(a / a_{0}\right)^{-n}$. Setting $P=P_{0}\left(a / a_{0}\right)^{3 / 2}$, the collision time is then $t_{c}=t_{0}\left(a / a_{0}\right)^{3 / 2+p-n}$, where

$$
t_{0}=\frac{\alpha_{0} r_{\max } \rho P_{0}}{12 \pi x_{m} \Sigma_{0}}
$$

To derive the evolution of the disk, we assume the particles have a differential size distribution $N(r) \propto r^{-3.5}$ in each annulus (e.g., Dohnanyi 1969; Williams \& Wetherill 1994; Tanaka et al. 1996; Kobayashi \& Tanaka 2010). Other choices lead to similar results (Wyatt et al. 2011). We can then relate the cross-sectional area of the swarm to the mass in each annulus:

$$
d A=\frac{3}{4 \rho}\left(\frac{1}{r_{\min } r_{\max }}\right)^{1 / 2} d M
$$

Setting $d M=\Sigma_{s} 2 \pi a d a, \tilde{a}=a / a_{0}$ and $d \tilde{a}=d a / a_{0}$, the general result of the analytic model in eq. A2 and our relation for $t_{c}$ yields:

$$
d A(t)=\frac{3 \pi x_{m} \Sigma_{0} a_{0}^{2} \tilde{a}^{5 / 2-n} d \tilde{a}}{2 \rho}\left(\frac{1}{r_{\min } r_{\max }}\right)^{1 / 2}\left(\tilde{a}^{-n+p+3 / 2}+t / t_{0}\right)^{-1} .
$$

To relate this evolution to an observable quantity, we set the relative luminosity $d L(t) / L_{\star}=$ $d A(t) / 4 \pi a^{2}=d A(t) / 4 \pi \tilde{a}^{2} a_{0}^{2}$. We then have:

$$
\frac{d L(t)}{L_{\star}}=\frac{3 x_{m} \Sigma_{0} \tilde{a}^{1 / 2-n} d \tilde{a}}{8 \rho}\left(\frac{1}{r_{\min } r_{\max }}\right)^{1 / 2}\left(\tilde{a}^{-n+p+3 / 2}+t / t_{0}\right)^{-1} .
$$

Integrating eqs. A6 A7) over $\tilde{a}$ yields the time evolution of the cross-sectional area and relative luminosity for material between $\tilde{a}_{\text {in }}=a_{\text {in }} / a_{0}$ and $\tilde{a}_{\text {out }}=a_{\text {out }} / a_{0}$.

Applying this theory to a real disk requires specifying parameters for the particles $\left(r_{\min }\right.$, $r_{\max }$, and $\left.\rho\right)$ and the disk $\left(\alpha_{0}, n, x_{m}\right.$, and $\left.p\right)$. Radiation pressure from the central star ejects particles with radii smaller than $r_{\min }$ (Burns et al. 1979); here, we set $r_{\min }=1 \mu \mathrm{m}$. For

8 Kennedy \& Wyatt (2010) developed a semi-analytic theory for extended debris disks around A-type stars. For the evolution of IR excesses at specific wavelengths, our approach is similar; our analytic derivation for the evolution of the luminosity is new. 
equal mass objects with relative velocity $v \approx e v_{K}$, the collision energy is $Q_{c}=v^{2} / 8$. The binding energy is

$$
Q_{D}^{\star}=Q_{b} r^{\beta_{b}}+Q_{g} \rho_{p} r^{\beta_{g}}
$$

where $Q_{b} r^{\beta_{b}}$ is the bulk component of the binding energy and $Q_{g} \rho_{g} r^{\beta_{g}}$ is the gravity component of the binding energy (e.g., Benz \& Asphaug 1999; Leinhardt et al. 2008; Leinhardt \& Stewart 2009). Adopting parameters appropriate for rocky objects and setting $Q_{c} \approx Q_{D}^{\star}$ yields (KB2016):

$$
r_{c, \text { max }} \approx 300\left(\frac{e}{0.1}\right)^{1.48}\left(\frac{v_{K}}{30 \mathrm{~km} \mathrm{~s}^{-1}}\right)^{1.48}\left(\frac{\rho}{3 \mathrm{~g} \mathrm{~cm}^{-3}}\right)^{-0.74}\left(\frac{Q_{g}}{0.3 \mathrm{erg} \mathrm{g}^{-1}}\right)^{-0.74} \mathrm{~km}
$$

Based on several simulations of planet formation in the terrestrial zone, $e \approx 0.1$ is a reasonable choice (e.g., Weidenschilling et al. 1997; Kenyon \& Bromley 2006; Raymond et al. 2007; Chambers 2013). Setting $r_{\max }=300 \mathrm{~km}$, the collision time at $1 \mathrm{AU}$ is:

$$
t_{0} \approx 2.4 \times 10^{5} \alpha_{0} x_{m}^{-1}\left(\frac{r_{\max }}{300 \mathrm{~km}}\right)\left(\frac{\Sigma_{0}}{10 \mathrm{~g} \mathrm{~cm}^{-2}}\right)^{-1}\left(\frac{P_{0}}{1 \mathrm{yr}}\right) \mathrm{yr} .
$$

For a standard protostellar disk with $\Sigma_{s} \propto a^{-p}$, we adopt $p=3 / 2$ (e.g. Birnstiel et al. 2010; Windmark et al. 2012; Testi et al. 2014). In the standard analytic model, $\alpha \propto\left(v^{2} / Q_{D}^{\star}\right)^{-n}$ with $n=5 / 6$ (Wyatt et al. 2007a b; Kobayashi \& Tanaka 2010). However, comprehensive numerical simulations suggest $n=1$ (KB2016). Here, we consider $\alpha_{0}=1$ and $n=0$ ( $v=$ constant throughout the disk $)$ or $n=1$ ( $e=$ constant throughout the disk $)$. Adopting $\tilde{t}=t / t_{0}$, the luminosity evolution is then

$$
\frac{L(t)}{L_{\star}}=\frac{x_{m} \Sigma_{0}}{4 \rho}\left(\frac{1}{r_{\min } r_{\max }}\right)^{1 / 2}\left[\tan ^{-1}\left(\frac{\tilde{a}_{\text {out }}^{3 / 2}}{\sqrt{\tilde{t}}}\right)-\tan ^{-1}\left(\frac{\tilde{a}_{\text {in }}^{3 / 2}}{\sqrt{\tilde{t}}}\right)\right] \tilde{t}^{-1 / 2},
$$

for $n=0$ and

$$
\frac{L(t)}{L_{\star}}=\frac{3 \sqrt{2} x_{m} \Sigma_{0}}{32 \rho}\left(\frac{1}{r_{\min } r_{\max }}\right)^{1 / 2} \tilde{t}^{-3 / 4} \Lambda(\tilde{a}, \tilde{t})
$$

for $n=1$, where

$$
\Lambda(\tilde{a}, \tilde{t})=\left[\ln \left(\frac{\tilde{a}+\tilde{t}^{1 / 2}+\sqrt{2 \tilde{a} \tilde{t}^{1 / 2}}}{\tilde{a}+\tilde{t}^{1 / 2}-\sqrt{2 \tilde{a} \tilde{t}^{1 / 2}}}\right)+2 \tan ^{-1}\left(\sqrt{2 \tilde{a} / \tilde{t}^{1 / 2}}+1\right)+2 \tan ^{-1}\left(\sqrt{2 \tilde{a} / \tilde{t}^{1 / 2}}-1\right)\right]_{\tilde{a}_{\text {in }}}^{\tilde{a}_{\text {out }}} .
$$

At $t=0$, solutions for any $n$ yield the same initial luminosity:

$$
\frac{L_{\text {init }}}{L_{\star}}=\frac{x_{m} \Sigma_{0}}{4 \rho}\left(\frac{1}{r_{\min } r_{\max }}\right)^{1 / 2}\left(\tilde{a}_{\text {in }}^{-3 / 2}-\tilde{a}_{\text {out }}^{-3 / 2}\right)
$$


In terms of our adopted parameters:

$$
\frac{L_{\text {init }}}{L_{\star}} \approx 0.5 x_{m}\left(\frac{r_{\min }}{1 \mu \mathrm{m}}\right)^{-1 / 2}\left(\frac{r_{\max }}{300 \mathrm{~km}}\right)^{-1 / 2}\left(\frac{a_{\text {in }}}{0.1 \mathrm{AU}}\right)^{-3 / 2}\left[1-\left(\frac{a_{\text {in }}}{a_{\text {out }}}\right)^{3 / 2}\right] .
$$

When $x_{m} \gtrsim 2 \times 10^{-4}$, the initial luminosity of the debris disk exceeds our nominal detection limit, $L_{d} / L_{\star} \gtrsim 10^{-4}$. At late times, we derive a single expression when $n=0$ or $n=1$ :

$$
\frac{L_{d}\left(t \gg t_{0}\right)}{L_{\star}} \simeq \frac{(2 n+1) x_{m} \Sigma_{0}}{4 \rho}\left(\tilde{a}_{\text {out }}^{-n+3 / 2}-\tilde{a}_{\text {in }}^{-n+3 / 2}\right)\left(\frac{1}{r_{\min } r_{\max }}\right)^{1 / 2} \tilde{t}^{-1} .
$$

As in the standard model for a single annulus, the luminosity declines linearly with time when $t \gg t_{0}$. For our adopted parameters and $n=0$ :

$$
\frac{L_{d}\left(t \gg t_{0}\right)}{L_{\star}} \approx 0.04 x_{m}\left(\frac{r_{\min }}{1 \mu \mathrm{m}}\right)^{-1 / 2}\left(\frac{r_{\max }}{300 \mathrm{~km}}\right)^{-1 / 2}\left(\frac{a_{\text {out }}}{2 \mathrm{AU}}\right)^{-3 / 2}\left[1-\left(\frac{a_{\text {out }}}{a_{\text {in }}}\right)^{3 / 2}\right] \tilde{t}^{-1} .
$$

With $x_{m}=1$ and $t=10 \mathrm{Myr}, L_{d} / L_{\star} \approx 10^{-3}$. The relative luminosity reaches the nominal detection limit of $L_{d} / L_{\star} \approx 10^{-4}$ at roughly $100 \mathrm{Myr}$.

Fig. 7 illustrates results for a disk with $a_{\text {in }}=0.1 \mathrm{AU}$ and $a_{\text {out }}=2 \mathrm{AU}$. The two sets of curves compare the analytic model with numerical results derived from dividing the disk into 101 annuli and summing $d L(t) / L_{\star}$ (eq. A7) from each annulus. There is superb agreement between the two approaches. For any time, the difference between the analytic and numerical results for $n=0$ or $n=1$ is less than $0.1 \%$. At $10 \mathrm{Myr}$, all of the model curves lie above the approximate detection limit of $L_{d} / L_{\star} \approx 10^{-4}$.

To derive the predicted evolution of disk fluxes at specific wavelengths, we assume all particles radiate as blackbodies at the equilibrium temperature, $d F(t)=d A(t) B_{\nu}(T)$, where $T=280\left(a / a_{0}\right)^{-1 / 2} \mathrm{~K}$. Although $d F(t)$ might be integrable, the result is very messy. Given the good agreement between the analytical and numerical results for $L_{d}(t) / L_{\star}$, we integrate $d F(t)$ numerically.

\section{A.2. Gas Drag in a Depleted Disk}

As outlined in the main text, drag within a remnant gaseous disk can rapidly remove small particles from a debris disk around 10 Myr old stars. To quantify this drift, we adopt a model gaseous disk (e.g., Adachi et al. 1976b; Weidenschilling 1977a; Kenyon \& Hartmann 1987; Chiang \& Goldreich 1997; Takeuchi \& Artymowicz 2001; Rafikov 2004; Chiang \& Youdin 2010; Youdin \& Kenyon 2013; Armitage 2013) with surface density, 
$\Sigma_{g}=\Sigma_{g, 0}(a / 1 \mathrm{AU})^{-p}$, midplane temperature $T_{g}=T_{g, 0}(a / 1 \mathrm{AU})^{-q}$, and vertical scale height $H / a=H_{0}(a / 1 \mathrm{AU})^{s}$. The midplane density is $\rho_{g}=\Sigma_{g} / 2 H$. Setting the sound speed $c_{s}^{2}=\left(\gamma k_{B} T_{g} / \mu m_{H}\right)$ - where $\gamma$ is the ratio of specific heats, $k_{B}$ is Boltzmann's constant, $\mu$ is the mean molecular weight, and $m_{H}$ is the mass of a hydrogen atom, the gas pressure in the midplane is $P_{g}=\rho c_{s}^{2} / \gamma$. The gas has mean-free-path $\lambda=\mu m_{H} c_{s} / \Omega \Sigma_{g} \sigma_{H_{2}}$ and viscosity $\nu=\lambda v_{t} \rho_{g} / 3$, where $\sigma_{H_{2}} \simeq 10^{-15} \mathrm{~cm}^{2}$ is the collision cross-section for $\mathrm{H}_{2}$, and $v_{t}^{2}=\left(8 k_{B} T_{g} / \pi \mu m_{H}\right)$ is the thermal velocity.

For the calculations in this paper we adopt $p=1, q=0.5, H_{0}=0.03, s=1 / 8, \gamma=1.4$, and $\mu=2.0$. Other choices for the exponents $-p=0.5-1.5, q=0.3-0.7$, and $s=0.0-0.3$ - have modest impact on the results. In all configurations, the ratio of the mass density of solids to the mass density of gas is small.

Particles with radii $r \leq 9 \lambda / 4$ are in the Epstein regime, where the drag force is $F_{d}=$ $\pi r^{2} \rho v_{d}\left(v_{t}^{2}+v_{d}^{2}\right)^{1 / 2}$. As in Takeuchi \& Artymowicz (2001), the term $\left(v_{t}^{2}+v_{d}^{2}\right)^{1 / 2}$ provides an estimate of the drag force in the subsonic and supersonic regimes. For larger particles, all drift is in the subsonic regime, where the drag force is $F_{d}=C_{d} \pi r^{2} \rho v_{d}^{2} / 2$ and $C_{d}$ is a drag coefficient which depends on the Reynolds number $R e=2 \rho v_{d} r / \nu$ (e.g., eqs. $8 \mathrm{a}-8 \mathrm{c}$ in Weidenschilling 1977a).

The main parameters in this model are the gravity of the central star $g=G M_{\star} / a^{2}$, the residual gravity $\Delta g=\rho_{g}^{-1} d P / d a$, and the stopping time $t_{s}=m v_{d} / F_{d}$ (Adachi et al. 1976a; Weidenschilling 1977a; Takeuchi \& Artymowicz 2001). The gas orbits at a slightly smaller velocity than the local circular velocity. Defining $\eta=-\Delta g / g$, the gas velocity is

$$
v_{g}=v_{K}(1-\eta)^{1 / 2} \text {. }
$$

The maximum velocity of drifting particles is roughly:

$$
v_{d, \max } \approx \eta v_{K} / 2
$$

In an optically thin disk, radiation pressure drives small particles radially outward (e.g., Burns et al. 1979; Takeuchi \& Artymowicz 2001). Defining $\beta$ as the ratio of the radiation pressure force to the gravitational force:

$$
\beta=\frac{3 L_{\star} Q_{p r}}{16 \pi c G M_{\star} r \rho_{s}},
$$

where $c$ is the speed of light. Although Takeuchi \& Artymowicz (2001) also consider the impact of Poynting-Robertson drag, this drag has little impact when the collision time is short. Thus, we ignore Poynting-Robertson drag in this discussion. 
Weidenschilling (1977a) and Takeuchi \& Artymowicz (2001) divide the drift velocity of solid particles relative to the gas into a radial component $v_{\text {rad }}$ and an azimuthal component $v_{\theta}$, with $v_{d}^{2}=v_{r a d}^{2}+v_{\theta}^{2}$. Defining the angular velocity $\Omega=\left(G M_{\star} / a^{3}\right)^{1 / 2}$ and setting $\tau_{s}=t_{s} \Omega$, the two components of the drift are

$$
v_{\text {rad }}=(\eta-\beta) v_{K} /\left(\tau_{s}+\tau_{s}^{-1}\right)
$$

and

$$
v_{\theta}=\tau_{s} v_{\text {rad }} / 2
$$

Three parameters $-\beta, \eta$, and $\tau_{s}$ - divide drift into four major regimes. Large particles poorly-coupled to the gas $\left(\tau_{s} \gg 1\right)$ follow circular orbits with $v_{\text {rad }} \approx 0$ and $v_{\theta} \approx \eta v_{K}$. Intermediate size particles weakly coupled to the gas $\left(\tau_{s} \approx 1\right)$ have maximal drift velocities and somewhat smaller angular velocities relative to the gas. Small particles $\left(\tau_{s} \ll 1\right)$ are entrained in the gas. When $\eta \gtrsim \beta(\eta \lesssim \beta)$ for small objects, $v_{\text {rad }}$ is positive (negative); particles drift inward (outward).

To identify these regimes in gaseous disks at $1 \mathrm{AU}$, we develop an iterative technique to solve eqs. A21 A22). For each particle size, the algorithm adopts a drag regime, derives $t_{s}$ and $\tau_{s}$, infers $v_{\text {rad }}$ and $v_{\theta}$, and then verifies the drag regime. This process repeats until the final drag regime is identical to the initial drag regime. When particles are small enough to experience Epstein drag, the algorithm loops through an initial iteration to derive a consistent $v_{d}$ for the subsonic and supersonic regimes. Comparisons with results in Weidenschilling (1977a) and Takeuchi \& Artymowicz (2001) confirm the accuracy of our approach.

Fig. 8 illustrates the variation in drift velocity with semimajor axis for particles with sizes of $1-100 \mu \mathrm{m}$ in a gaseous disk with a surface density of $0.001 \%$ of the MMSN. Close to the star, radiation pressure drives particles to larger $a$ (open circles). Far from the star, the gas drags particles inward (filled circles). At some intermediate $a$, small particles find an equilibrium distance $a_{e q}$ where radiation pressure balances gas drag. For the disk model adopted here, this equilibrium is at

$$
a_{e q} \approx \frac{40 \mu \mathrm{m}}{r} \mathrm{AU}
$$

In solar-type stars with $r_{\text {min }} \approx 1 \mu \mathrm{m}$, small particles with $r \approx 1-10 \mu \mathrm{m}$ produce most of the IR excess. When the disk's surface density is $0.001 \%$ of the MMSN, these small particles have $a_{e q} \gtrsim 4 \mathrm{AU}$ and $T_{d} \lesssim 140 \mathrm{~K}$. These particles are then too cold to produce a warm debris disk. 


\section{REFERENCES}

Adachi, I., Hayashi, C., \& Nakazawa, K. 1976a, Progress of Theoretical Physics, 56, 1756

—. 1976b, Progress of Theoretical Physics, 56, 1756

Agnor, C., \& Asphaug, E. 2004, ApJ, 613, L157

Agnor, C. B., Canup, R. M., \& Levison, H. F. 1999, Icarus, 142, 219

Airapetian, V. S., \& Usmanov, A. V. 2016, ApJ, 817, L24

Alexander, R., Pascucci, I., Andrews, S., Armitage, P., \& Cieza, L. 2014, in Protostars and Planets VI, ed. H. Beuther, R. S. Klessen, C. P. Dullemond, \& T. Henning (University of Arizona Press, Tucson, AZ), 475-496

ALMA Partnership, Brogan, C. L., Pérez, L. M., et al. 2015, ApJ, 808, L3

Andrews, S. M. 2015, PASP, 127, 961

Armitage, P. J. 2003, ApJ, 582, L47

—. 2013, Astrophysics of Planet Formation (Cambridge University Press, Cambridge, UK)

Asphaug, E., Agnor, C. B., \& Williams, Q. 2006, Nature, 439, 155

Backman, D. E., \& Paresce, F. 1993, in Protostars and Planets III, ed. E. H. Levy \& J. I. Lunine, 1253-1304

Ballering, N. P., Rieke, G. H., Su, K. Y. L., \& Montiel, E. 2013, ApJ, 775, 55

Baraffe, I., Chabrier, G., Allard, F., \& Hauschildt, P. H. 1998, A\&A, 337, 403

Baraffe, I., Homeier, D., Allard, F., \& Chabrier, G. 2015, A\&A, 577, A42

Batalha, N. M., Rowe, J. F., Bryson, S. T., et al. 2013, ApJS, 204, 24

Beichman, C. A., Lisse, C. M., Tanner, A. M., et al. 2011, ApJ, 743, 85

Benz, W., \& Asphaug, E. 1999, Icarus, 142, 5

Beust, H., Vidal-Madjar, A., Ferlet, R., \& Lagrange-Henri, A. M. 1990, A\&A, 236, 202

Birnstiel, T., Dullemond, C. P., \& Brauer, F. 2010, A\&A, 513, A79+

Birnstiel, T., Fang, M., \& Johansen, A. 2016, ArXiv e-prints, arXiv:1604.02952 
Bizzarro, M., Baker, J. A., Haack, H., \& Lundgaard, K. L. 2005, ApJ, 632, L41

Bouchet, P., García-Marín, M., Lagage, P.-O., et al. 2015, PASP, 127, 612

Bressan, A., Marigo, P., Girardi, L., et al. 2012, MNRAS, 427, 127

Bromley, B. C., \& Kenyon, S. J. 2006, AJ, 131, 2737

-. 2011, ApJ, 735, 29

Buchhave, L. A., Bizzarro, M., Latham, D. W., et al. 2014, Nature, 509, 593

Burke, C. J., Christiansen, J. L., Mullally, F., et al. 2015, ApJ, 809, 8

Burns, J. A., Lamy, P. L., \& Soter, S. 1979, Icarus, 40, 1

Campo Bagatin, A., Cellino, A., Davis, D. R., Farinella, P., \& Paolicchi, P. 1994, Planet. Space Sci., 42, 1079

Canup, R. M., \& Asphaug, E. 2001, Nature, 412, 708

Carpenter, J. M., Mamajek, E. E., Hillenbrand, L. A., \& Meyer, M. R. 2006, ApJ, 651, L49

-. 2009a, ApJ, 705, 1646

Carpenter, J. M., Bouwman, J., Mamajek, E. E., et al. 2009b, ApJS, 181, 197

Cataldi, G., Brandeker, A., Olofsson, G., et al. 2014, A\&A, 563, A66

Chambers, J. 2008, Icarus, 198, 256

Chambers, J. E. 2001, Icarus, 152, 205

-. 2013, Icarus, 224, 43

-. 2014, Icarus, 233, 83

-. 2016, ApJ, 825, 63

Chambers, J. E., \& Wetherill, G. W. 1998, Icarus, 136, 304

Chambers, J. E., Wetherill, G. W., \& Boss, A. P. 1996, Icarus, 119, 261

Chen, C. H., Mamajek, E. E., Bitner, M. A., et al. 2011, ApJ, 738, 122

Chen, Y., Girardi, L., Bressan, A., et al. 2014, MNRAS, 444, 2525 
Chiang, E., \& Laughlin, G. 2013, MNRAS, 431, 3444

Chiang, E., \& Youdin, A. N. 2010, Annual Review of Earth and Planetary Sciences, 38, 493

Chiang, E. I., \& Goldreich, P. 1997, ApJ, 490, 368

Cloutier, R., Currie, T., Rieke, G. H., et al. 2014, ApJ, 796, 127

Cohen, O., \& Drake, J. J. 2014, ApJ, 783, 55

Colón, K. D., Morehead, R. C., \& Ford, E. B. 2015, MNRAS, 452, 3001

Coughlin, J. L., Mullally, F., Thompson, S. E., et al. 2016, ApJS, 224, 12

Cuello, N., Gonzalez, J.-F., \& Pignatale, F. C. 2016, MNRAS, 458, 2140

Cumming, A., Butler, R. P., Marcy, G. W., et al. 2008, PASP, 120, 531

Currie, T., Kenyon, S. J., Rieke, G., Balog, Z., \& Bromley, B. C. 2007, ApJ, 663, L105

Currie, T., Lisse, C. M., Sicilia-Aguilar, A., Rieke, G. H., \& Su, K. Y. L. 2011, ApJ, 734, 115

Czechowski, A., \& Mann, I. 2007, ApJ, 660, 1541

Dahm, S. E., \& Carpenter, J. M. 2009, AJ, 137, 4024

Dauphas, N., \& Chaussidon, M. 2011, Annual Review of Earth and Planetary Sciences, 39, 351

Dauphas, N., \& Pourmand, A. 2011, Nature, 473, 489

David, T. J., Hillenbrand, L. A., Petigura, E. A., et al. 2016, Nature, 534, 658

Davis, D. R., Chapman, C. R., Weidenschilling, S. J., \& Greenberg, R. 1985, Icarus, 63, 30

Davis, D. R., \& Ryan, E. V. 1990, Icarus, 83, 156

Dent, W. R. F., Walker, H. J., Holland, W. S., \& Greaves, J. S. 2000, MNRAS, 314, 702

Désert, J.-M., Charbonneau, D., Torres, G., et al. 2015, ApJ, 804, 59

Dohnanyi, J. S. 1969, J. Geophys. Res., 74, 2531

Dominik, C., \& Decin, G. 2003, ApJ, 598, 626

Donati, J. F., Moutou, C., Malo, L., et al. 2016, Nature, 534, 662 
Doppmann, G. W., Najita, J. R., \& Carr, J. S. 2016, ApJ, submitted

Eiroa, C., Marshall, J. P., Mora, A., et al. 2013, A\&A, 555, A11

Fabrycky, D. C., Lissauer, J. J., Ragozzine, D., et al. 2014, ApJ, 790, 146

Fang, J., \& Margot, J.-L. 2012, ApJ, 761, 92

-. 2013, ApJ, 767, 115

Fedele, D., van den Ancker, M. E., Henning, T., Jayawardhana, R., \& Oliveira, J. M. 2010, A\&A, 510, A72

Ferlet, R., Vidal-Madjar, A., \& Hobbs, L. M. 1987, A\&A, 185, 267

Foreman-Mackey, D., Hogg, D. W., \& Morton, T. D. 2014, ApJ, 795, 64

Foreman-Mackey, D., Montet, B. T., Hogg, D. W., et al. 2015, ApJ, 806, 215

Fressin, F., Torres, G., Charbonneau, D., et al. 2013, ApJ, 766, 81

Gaidos, E., Mann, A. W., RIzzuto, A., et al. 2016, ArXiv e-prints, arXiv:1606.05812

Garaud, P., Meru, F., Galvagni, M., \& Olczak, C. 2013, ApJ, 764, 146

Genda, H., Fujita, T., Kobayashi, H., Tanaka, H., \& Abe, Y. 2015a, Icarus, 262, 58

Genda, H., Kobayashi, H., \& Kokubo, E. 2015b, ApJ, 810, 136

Genda, H., Kokubo, E., \& Ida, S. 2012, ApJ, 744, 137

Goldreich, P., Lithwick, Y., \& Sari, R. 2004, ARA\&A, 42, 549

Gorti, U., \& Hollenbach, D. 2004, ApJ, 613, 424

Gorti, U., Hollenbach, D., \& Dullemond, C. P. 2015, ApJ, 804, 29

Gould, A., Udalski, A., An, D., et al. 2006, ApJ, 644, L37

Greaves, J. S., \& Rice, W. K. M. 2010, MNRAS, 407, 1981

Greenberg, R., Hartmann, W. K., Chapman, C. R., \& Wacker, J. F. 1978, Icarus, 35, 1

Grogan, K., Dermott, S. F., \& Durda, D. D. 2001, Icarus, 152, 251

Gustafson, B. A. S. 1994, Annual Review of Earth and Planetary Sciences, 22, 553 
Haisch, Jr., K. E., Lada, E. A., \& Lada, C. J. 2001, ApJ, 553, L153

Hansen, B. M. S. 2015, International Journal of Astrobiology, 14, 267

Hansen, B. M. S., \& Murray, N. 2012, ApJ, 751, 158

-. 2013, ApJ, 775, 53

Hartmann, L., Calvet, N., Gullbring, E., \& D’Alessio, P. 1998, ApJ, 495, 385

Hayashi, C. 1981, Progress of Theoretical Physics Supplement, 70, 35

Heng, K., \& Tremaine, S. 2010, MNRAS, 401, 867

Herrmann, F., \& Krivov, A. V. 2007, A\&A, 476, 829

Hillenbrand, L. A., Carpenter, J. M., Kim, J. S., et al. 2008, ApJ, 677, 630

Hobbs, L. M., Vidal-Madjar, A., Ferlet, R., Albert, C. E., \& Gry, C. 1985, ApJ, 293, L29

Holsapple, K., Giblin, I., Housen, K., Nakamura, A., \& Ryan, E. 2002, in Asteroids III, ed. W. F. Bottke, A. Cellino, P. Paolicchi, \& R. P. Binzel (University of Arizona Press, Tucson, AZ), 443-462

Housen, K. R., \& Holsapple, K. A. 1990, Icarus, 84, 226

-. 1999, Icarus, 142, 21

Howell, S. B., Sobeck, C., Haas, M., et al. 2014, PASP, 126, 398

Ida, S., Bryden, G., Lin, D. N. C., \& Tanaka, H. 2000, ApJ, 534, 428

Ida, S., \& Lin, D. N. C. 2008, ApJ, 673, 487

Ingleby, L., Calvet, N., Hernández, J., et al. 2011a, AJ, 141, 127

—. 2014, ApJ, 790, 47

Ingleby, L., Calvet, N., Bergin, E., et al. 2011b, ApJ, 743, 105

Jackson, A. P., \& Wyatt, M. C. 2012, MNRAS, 425, 657

Johansen, A., Mac Low, M.-M., Lacerda, P., \& Bizzarro, M. 2015, Science Advances, 1, 15109

Johansen, A., Oishi, J. S., Mac Low, M.-M., et al. 2007, Nature, 448, 1022 
Johns-Krull, C. M., McLane, J. N., Prato, L., et al. 2016a, ArXiv e-prints, arXiv:1605.07917

Johns-Krull, C. M., Prato, L., McLane, J. N., et al. 2016b, ArXiv e-prints, arXiv:1606.02701

Kennedy, G. M., \& Kenyon, S. J. 2009, ApJ, 695, 1210

Kennedy, G. M., \& Wyatt, M. C. 2010, MNRAS, 405, 1253

-. 2012, MNRAS, 426, 91

-. 2013, MNRAS, 433, 2334

Kenyon, S. J., \& Bromley, B. C. 2002, ApJ, 577, L35

—. 2004, ApJ, 602, L133

-. 2005, AJ, 130, 269

—. 2006, AJ, 131, 1837

—. 2008, ApJS, 179, 451

-. 2014, ApJ, 780, 4

—. 2016, ApJ, 817, 51

Kenyon, S. J., Currie, T., \& Bromley, B. C. 2014, ApJ, 786, 70

Kenyon, S. J., Gómez, M., \& Whitney, B. A. 2008, in Handbook of Star Forming Regions, Volume I, ed. Reipurth, B. (San Francisco: Astronomical Society of the Pacific), $405-458$

Kenyon, S. J., \& Hartmann, L. 1987, ApJ, 323, 714

Kenyon, S. J., \& Luu, J. X. 1999, AJ, 118, 1101

Kiefer, F., Lecavelier des Etangs, A., Augereau, J.-C., et al. 2014, A\&A, 561, L10

Kleine, T., Touboul, M., Bourdon, B., et al. 2009, Geochim. Cosmochim. Acta, 73, 5150

Kobayashi, H., \& Tanaka, H. 2010, Icarus, 206, 735

Kokubo, E., \& Genda, H. 2010, ApJ, 714, L21

Kokubo, E., Kominami, J., \& Ida, S. 2006, ApJ, 642, 1131

Kominami, J., \& Ida, S. 2002, Icarus, 157, 43 
-. 2004, Icarus, 167, 231

Kral, Q., Wyatt, M., Carswell, R. F., et al. 2016, MNRAS, 461, 845

Krauss, O., \& Wurm, G. 2005, ApJ, 630, 1088

Kuchner, M. J. 2004, ApJ, 612, 1147

Lagrange, A.-M., Backman, D. E., \& Artymowicz, P. 2000, in Protostars and Planets IV, ed. Mannings, V., Boss, A.P., Russell, S. S. (University of Arizona Press, Tucson, AZ), 639

Lagrange, A. M., Ferlet, R., \& Vidal-Madjar, A. 1987, A\&A, 173, 289

Leinhardt, Z. M., Dobinson, J., Carter, P. J., \& Lines, S. 2015, ApJ, 806, 23

Leinhardt, Z. M., \& Richardson, D. C. 2005, ApJ, 625, 427

Leinhardt, Z. M., \& Stewart, S. T. 2009, Icarus, 199, 542

—. 2012, ApJ, 745, 79

Leinhardt, Z. M., Stewart, S. T., \& Schultz, P. H. 2008, in The Solar System Beyond Neptune, ed. Barucci, M. A., Boehnhardt, H., Cruikshank, D. P., \& Morbidelli, A. (University of Arizona Press, Tucson, AZ), 195-211

Levison, H. F., Kretke, K. A., Walsh, K. J., \& Bottke, W. F. 2015, Proceedings of the National Academy of Science, 112, 14180

Lewis, J. S. 1972, Icarus, 16, 241

Limbach, M. A., \& Turner, E. L. 2015, Proceedings of the National Academy of Science, 112,20

Lisse, C. M., Beichman, C. A., Bryden, G., \& Wyatt, M. C. 2007, ApJ, 658, 584

Lisse, C. M., Chen, C. H., Wyatt, M. C., \& Morlok, A. 2008, ApJ, 673, 1106

Lisse, C. M., VanCleve, J., Adams, A. C., et al. 2006, Science, 313, 635

Luhman, K. L., \& Mamajek, E. E. 2012, ApJ, 758, 31

Lunine, J. I., O'brien, D. P., Raymond, S. N., et al. 2011, Advanced Science Letters, 4, 325

Macintosh, B., Graham, J. R., Ingraham, P., et al. 2014, Proceedings of the National Academy of Science, 111, 12661 
Mamajek, E. E. 2009, in American Institute of Physics Conference Series, Vol. 1158, American Institute of Physics Conference Series, ed. T. Usuda, M. Tamura, \& M. Ishii, $3-10$

Manara, C. F., Beccari, G., Da Rio, N., et al. 2013, A\&A, 558, A114

Mann, A. W., Newton, E. R., Rizzuto, A. C., et al. 2016, ArXiv e-prints, arXiv:1604.06165

Marcy, G. W., Isaacson, H., Howard, A. W., et al. 2014, ApJS, 210, 20

Masset, F. S., \& Papaloizou, J. C. B. 2003, ApJ, 588, 494

Matthews, B. C., Krivov, A. V., Wyatt, M. C., Bryden, G., \& Eiroa, C. 2014, in Protostars and Planet VI, ed. Beuther, H., Klessen, R. S., Dullemond, C. P., \& Henning, T. (The University of Arizona Press, Tucson, AZ), 521-544

Meyer, M. R., Hillenbrand, L. A., Backman, D., et al. 2006, PASP, 118, 1690

Montgomery, S. L., \& Welsh, B. Y. 2012, PASP, 124, 1042

Moór, A., Apai, D., Pascucci, I., et al. 2009, ApJ, 700, L25

Morbidelli, A., Lunine, J. I., O’Brien, D. P., Raymond, S. N., \& Walsh, K. J. 2012, Annual Review of Earth and Planetary Sciences, 40, 251

Morishima, R., Schmidt, M. W., Stadel, J., \& Moore, B. 2008, ApJ, 685, 1247

Morton, T. D., Bryson, S. T., Coughlin, J. L., et al. 2016, ApJ, 822, 86

Morton, T. D., \& Johnson, J. A. 2011, ApJ, 738, 170

Mullally, F., Coughlin, J. L., Thompson, S. E., et al. 2016, PASP, 128, 074502

—. 2015, ApJS, 217, 31

Muzerolle, J., Briceño, C., Calvet, N., et al. 2000, ApJ, 545, L141

Muzerolle, J., Hartmann, L., \& Calvet, N. 1998a, AJ, 116, 2965

-. 1998b, AJ, 116, 455

Najita, J., Carr, J. S., \& Mathieu, R. D. 2003, ApJ, 589, 931

Najita, J. R., Carr, J. S., Pontoppidan, K. M., et al. 2013, ApJ, 766, 134

Najita, J. R., \& Kenyon, S. J. 2014, MNRAS, 445, 3315 
Natta, A., Testi, L., Muzerolle, J., et al. 2004, A\&A, 424, 603

O'Brien, D. P., \& Greenberg, R. 2003, Icarus, 164, 334

O’Brien, D. P., Morbidelli, A., \& Levison, H. F. 2006, Icarus, 184, 39

Ohtsuki, K., Stewart, G. R., \& Ida, S. 2002, Icarus, 155, 436

Papaloizou, J. C. B., Nelson, R. P., Kley, W., Masset, F. S., \& Artymowicz, P. 2007, Protostars and Planets V, 655

Pascucci, I., Gorti, U., Hollenbach, D., et al. 2006, ApJ, 651, 1177

Patel, R. I., Metchev, S. A., \& Heinze, A. 2014, ApJS, 212, 10

Pecaut, M. J., Mamajek, E. E., \& Bubar, E. J. 2012, ApJ, 746, 154

Petigura, E. A., Howard, A. W., \& Marcy, G. W. 2013, Proceedings of the National Academy of Science, 110, 19273

Petrovich, C. 2015, ApJ, 808, 120

Preibisch, T., \& Mamajek, E. 2008, The Nearest OB Association: Scorpius-Centaurus (Sco OB2), ed. B. Reipurth (San Francisco: Astronomical Society of the Pacific), 235

Pu, B., \& Wu, Y. 2015, ApJ, 807, 44

Quintana, E. V., Barclay, T., Borucki, W. J., Rowe, J. F., \& Chambers, J. E. 2016, ApJ, 821,126

Rafikov, R. R. 2004, AJ, 128, 1348

Raymond, S. N., \& Cossou, C. 2014, MNRAS, 440, L11

Raymond, S. N., Kokubo, E., Morbidelli, A., Morishima, R., \& Walsh, K. J. 2014, Protostars and Planets VI, 595

Raymond, S. N., Quinn, T., \& Lunine, J. I. 2004, Icarus, 168, 1

-. 2005, ApJ, 632, 670

—. 2007, Astrobiology, 7, 66

Raymond, S. N., Armitage, P. J., Moro-Martín, A., et al. 2011, A\&A, 530, A62

-. 2012, A\&A, 541, A11 
Redfield, S. 2007, ApJ, 656, L97

Ribas, Á., Merín, B., Ardila, D. R., \& Bouy, H. 2012, A\&A, 541, A38

Ricker, G. R., Winn, J. N., Vanderspek, R., et al. 2015, Journal of Astronomical Telescopes, Instruments, and Systems, 1, 014003

Riviere-Marichalar, P., Elliott, P., Rebollido, I., et al. 2015, A\&A, 584, A22

Rizzuto, A. C., Ireland, M. J., Dupuy, T. J., \& Kraus, A. L. 2016, ApJ, 817, 164

Safronov, V. S. 1969, Evoliutsiia doplanetnogo oblaka. (Evolution of the Protoplanetary Cloud and Formation of the Earth and Planets, Nauka, Moscow [Translation 1972, NASA TT F-677] (1969.)

Salyk, C., Blake, G. A., Boogert, A. C. A., \& Brown, J. M. 2011, ApJ, 743, 112

Santerne, A., Díaz, R. F., Moutou, C., et al. 2012, A\&A, 545, A76

Santerne, A., Moutou, C., Tsantaki, M., et al. 2016, A\&A, 587, A64

Schulz, T., Münker, C., Palme, H., \& Mezger, K. 2009, Earth and Planetary Science Letters, 280,185

Sicilia-Aguilar, A., Hartmann, L. W., Fürész, G., et al. 2006, AJ, 132, 2135

Sicilia-Aguilar, A., Henning, T., \& Hartmann, L. W. 2010, ApJ, 710, 597

Siess, L., Dufour, E., \& Forestini, M. 2000, A\&A, 358, 593

Silburt, A., Gaidos, E., \& Wu, Y. 2015, ApJ, 799, 180

Silverstone, M. D., Meyer, M. R., Mamajek, E. E., et al. 2006, ApJ, 639, 1138

Simon, J. B., Armitage, P. J., Li, R., \& Youdin, A. N. 2016, ApJ, 822, 55

Sliski, D. H., \& Kipping, D. M. 2014, ApJ, 788, 148

Smith, R., Jeffries, R. D., \& Oliveira, J. M. 2011, MNRAS, 411, 2186

Stauffer, J. R., Rebull, L. M., Carpenter, J., et al. 2005, AJ, 130, 1834

Stauffer, J. R., Rebull, L. M., James, D., et al. 2010, ApJ, 719, 1859

Stewart, S. T., \& Leinhardt, Z. M. 2012, ApJ, 751, 32 
Sugiura, N., \& Fujiya, W. 2014, Meteoritics and Planetary Science, 49, 772

Sullivan, P. W., Winn, J. N., Berta-Thompson, Z. K., et al. 2015, ApJ, 809, 77

Takeuchi, T., \& Artymowicz, P. 2001, ApJ, 557, 990

Tanaka, H., Inaba, S., \& Nakazawa, K. 1996, Icarus, 123, 450

Testi, L., Birnstiel, T., Ricci, L., et al. 2014, in Protostars and Planet VI, ed. Beuther, H., Klessen, R. S., Dullemond, C. P., \& Henning, T. (The University of Arizona Press, Tucson, AZ), 339-361

Tremaine, S. 2015, ApJ, 807, 157

Tremaine, S., \& Dong, S. 2012, AJ, 143, 94

Trilling, D. E., Bryden, G., Beichman, C. A., et al. 2008, ApJ, 674, 1086

Urban, L. E., Rieke, G., Su, K., \& Trilling, D. E. 2012, ApJ, 750, 98

Van Eylen, V., \& Albrecht, S. 2015, ApJ, 808, 126

Vanderburg, A., Latham, D. W., Buchhave, L. A., et al. 2016, ApJS, 222, 14

Vican, L., \& Schneider, A. 2014, ApJ, 780, 154

Volk, K., \& Gladman, B. 2015, ApJ, 806, L26

von Borstel, I., \& Blum, J. 2012, A\&A, 548, A96

Walsh, K. J., Morbidelli, A., Raymond, S. N., O’Brien, D. P., \& Mandell, A. M. 2011, Nature, 475, 206

Ward, W. R. 1997, Icarus, 126, 261

Weidenschilling, S. J. 1974, Icarus, 22, 426

-. 1977a, MNRAS, 180, 57

-. 1977b, Ap\&SS, 51, 153

-. 1997, Icarus, 127, 290

—. 2010, ApJ, 722, 1716 
Weidenschilling, S. J., Spaute, D., Davis, D. R., Marzari, F., \& Ohtsuki, K. 1997, Icarus, 128,429

Weiss, L. M., \& Marcy, G. W. 2014, ApJ, 783, L6

Wells, M., Lee, D., Oudenhuysen, A., et al. 2006, in Society of Photo-Optical Instrumentation Engineers (SPIE) Conference Series, Vol. 6265, Society of Photo-Optical Instrumentation Engineers (SPIE) Conference Series, 626514

Welsh, B. Y., Craig, N., Crawford, I. A., \& Price, R. J. 1998, A\&A, 338, 674

Wetherill, G. W. 1980, ARA\&A, 18, 77

Wetherill, G. W., \& Stewart, G. R. 1993, Icarus, 106, 190

White, R. J., \& Basri, G. 2003, ApJ, 582, 1109

Williams, D. R., \& Wetherill, G. W. 1994, Icarus, 107, 117

Williams, J. P., \& Cieza, L. A. 2011, ARA\&A, 49, 67

Windmark, F., Birnstiel, T., Ormel, C. W., \& Dullemond, C. P. 2012, A\&A, 544, L16

Winn, J. N., \& Fabrycky, D. C. 2015, ARA\&A, 53, 409

Wood, B. E., Müller, H.-R., Redfield, S., \& Edelman, E. 2014, ApJ, 781, L33

Wright, G. S., Bortoletto, F., Bruce, Jr., C. F., et al. 2003, in Society of Photo-Optical Instrumentation Engineers (SPIE) Conference Series, Vol. 4850, IR Space Telescopes and Instruments, ed. J. C. Mather, 493-503

Wurz, P. 2012, in Astrophysics and Space Science Library, Vol. 385, Astrophysics and Space Science Library, ed. I. Mann, N. Meyer-Vernet, \& A. Czechowski, 161

Wyatt, M. C. 2008, ARA\&A, 46, 339

Wyatt, M. C., Clarke, C. J., \& Booth, M. 2011, Celestial Mechanics and Dynamical Astronomy, 111, 1

Wyatt, M. C., \& Dent, W. R. F. 2002, MNRAS, 334, 589

Wyatt, M. C., Smith, R., Greaves, J. S., et al. 2007a, ApJ, 658, 569

Wyatt, M. C., Smith, R., Su, K. Y. L., et al. 2007b, ApJ, 663, 365 
Xu, S., Jura, M., Dufour, P., \& Zuckerman, B. 2016, ApJ, 816, L22

Yoshinaga, K., Kokubo, E., \& Makino, J. 1999, Icarus, 139, 328

Youdin, A. N. 2010, in EAS Publications Series, Vol. 41, EAS Publications Series, ed. T. Montmerle, D. Ehrenreich, \& A.-M. Lagrange, 187-207

Youdin, A. N. 2011a, ApJ, 731, 99

-. 2011b, ApJ, 742, 38

Youdin, A. N., \& Goodman, J. 2005, ApJ, 620, 459

Youdin, A. N., \& Kenyon, S. J. 2013, From Disks to Planets, ed. T. D. Oswalt, L. M. French, \& P. Kalas (Dordrecht: Springer Science \& Business Media), 1

Zhang, K., Blake, G. A., \& Bergin, E. A. 2015, ApJ, 806, L7

Zuckerman, B., Melis, C., Rhee, J. H., Schneider, A., \& Song, I. 2012, ApJ, 752, 58

Zuckerman, B., Rhee, J. H., Song, I., \& Bessell, M. S. 2011, ApJ, 732, 61

Zuckerman, B., \& Song, I. 2004, ApJ, 603, 738 


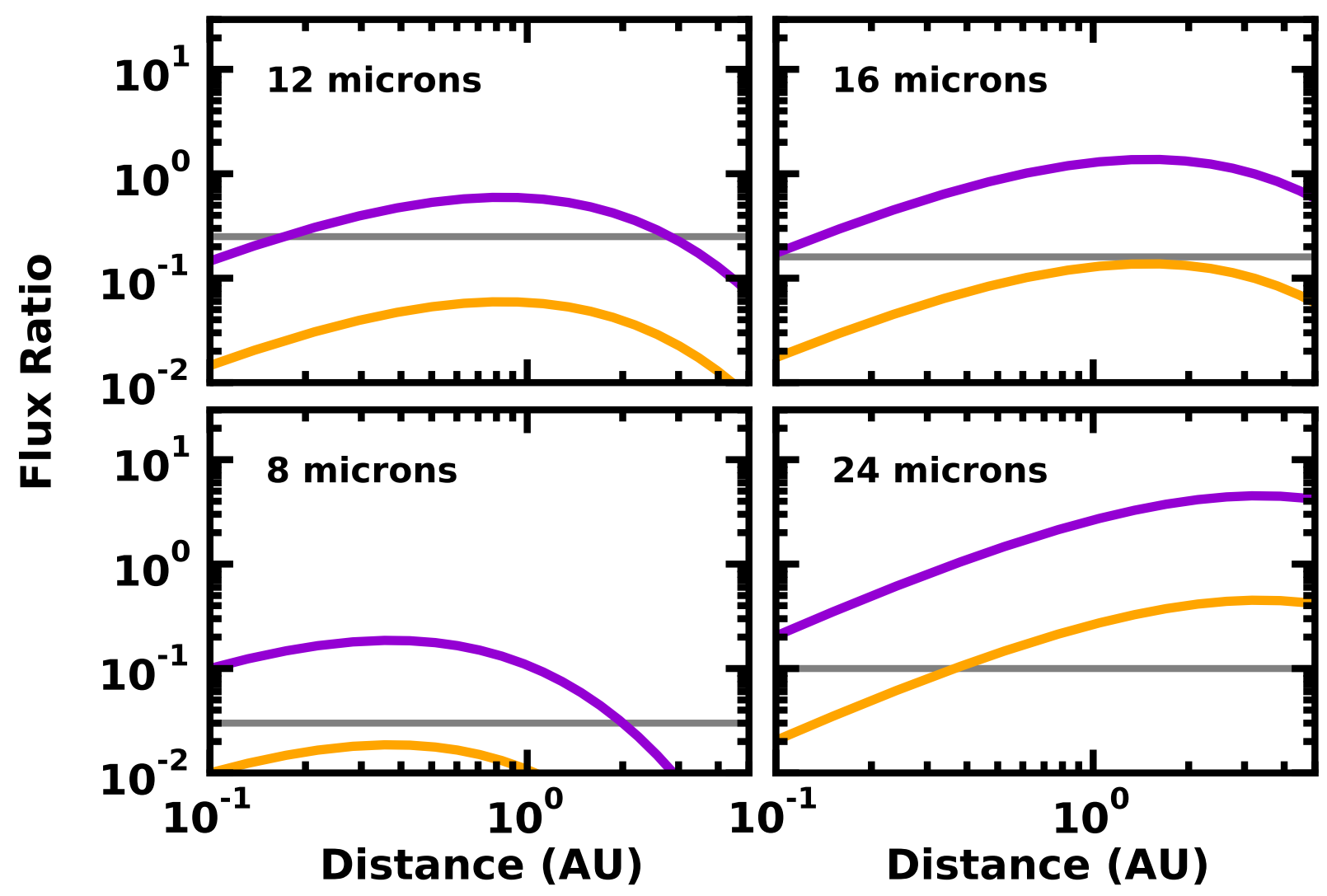

Fig. 1. - Predicted ratio of dust emission $F_{d}$ to photospheric emission $F_{\star}$ for material with temperature $T_{d}=280(r / 1 \mathrm{AU})^{-1 / 2} \mathrm{~K}$ and $L_{d} / L_{\star}=10^{-3}$ (violet curves) or $L_{d} / L_{\star}=10^{-4}$ (orange curves) orbiting a central star with $T_{\star}=5800 \mathrm{~K}$. Horizontal grey lines indicate typical detection limits from Spitzer $(8 \mu \mathrm{m}, 16 \mu \mathrm{m}$, and $24 \mu \mathrm{m}$; Carpenter et al. 2009a) and WISE (12 $\mu \mathrm{m}$; Luhman \& Mamajek 2012). 


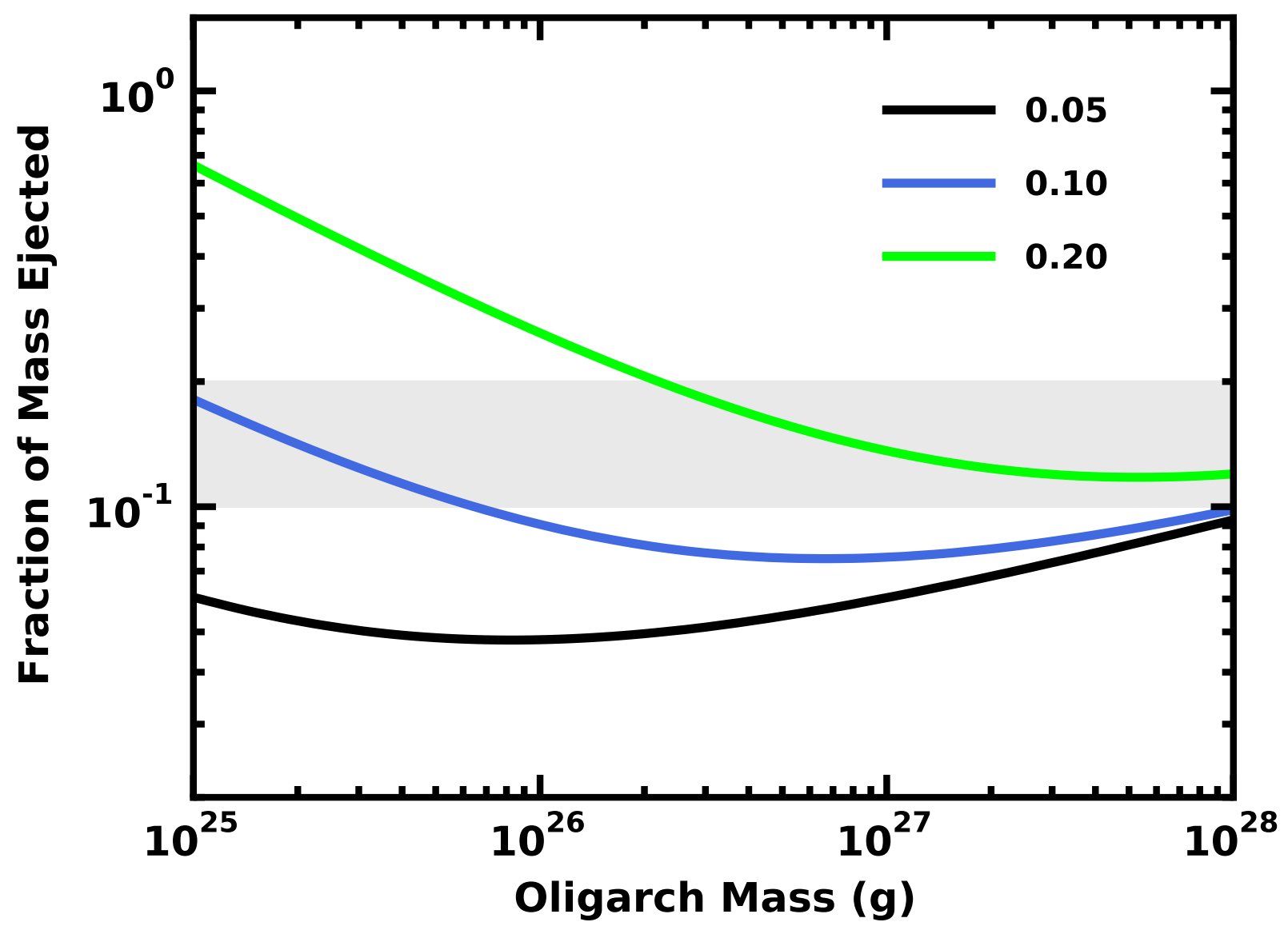

Fig. 2.- Predicted $f_{e j}=m_{e j} /\left(m_{1}+m_{2}\right)$ for collisions between equal mass oligarchs with $r$ $=1 \mathrm{AU}$, orbital eccentricity $e=0.05$ (black curve), 0.10 (blue curve), and 0.20 (green curve), and a binding energy $Q_{D}^{\star}$ appropriate for rocky objects in the terrestrial zone. For typical $e \sim 0.1$, collisions eject roughly $10 \%$ of the mass of the colliding pair of oligarchs. The grey bar indicates the range of $f_{e j}$ derived from several $n$-body simulations of protoplanets. 


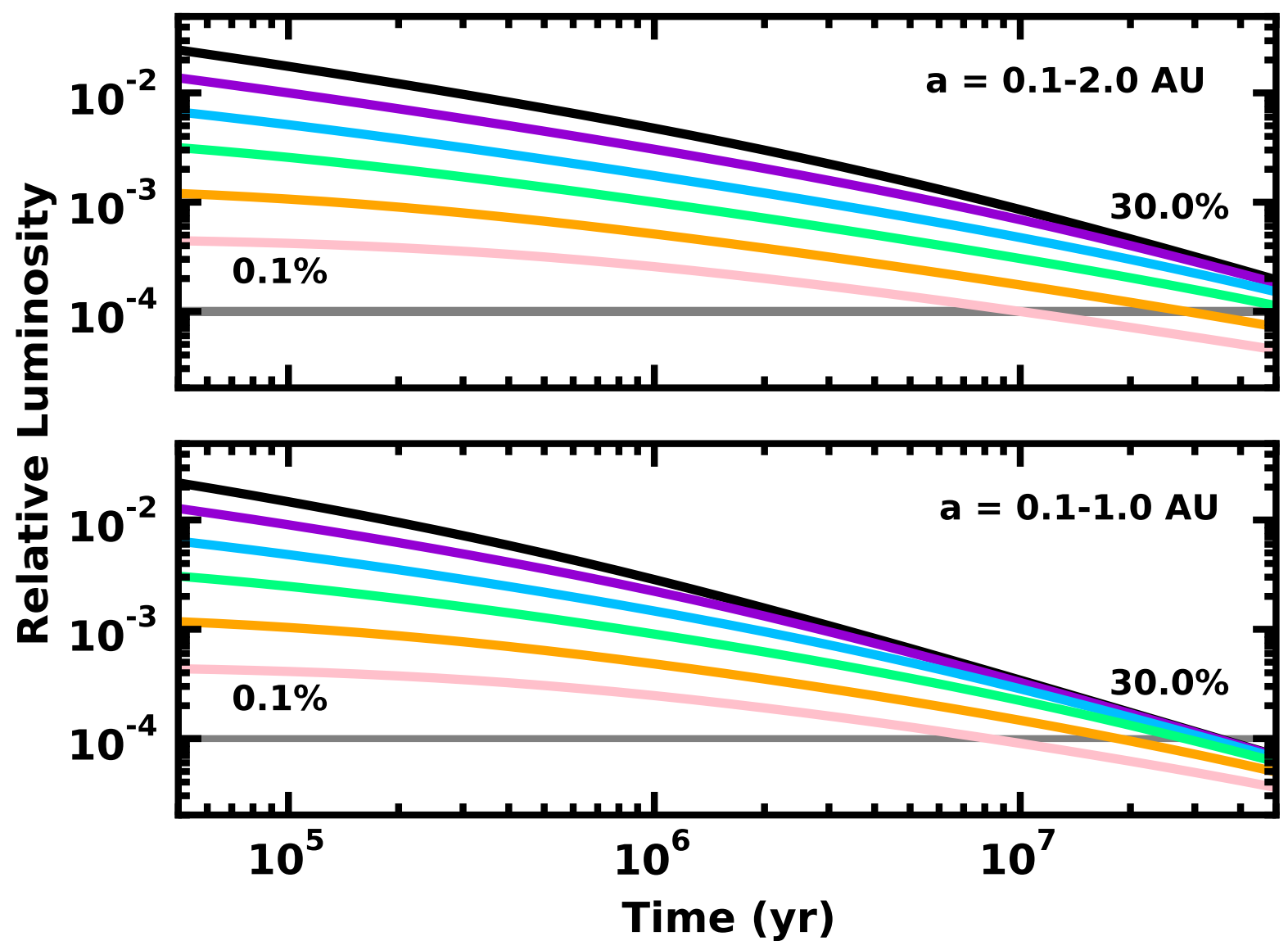

Fig. 3.- Time evolution of the luminosity ratio $L_{d} / L_{\star}$ for analytic models of debris disks at 0.1-1 AU (lower panel) and at 0.1-2 AU (upper panel). Curves plot results for models with $r_{\max }=300 \mathrm{~km}, e=0.1$, and $x_{m}=0.3$ (black), 0.1 (violet), 0.03 (blue), 0.01 (green), 0.003 (orange), and 0.001 (pink). Predicted luminosity ratios cross the nominal detection limit of $L_{d} / L_{\star}=10^{-4}$ at $10 \mathrm{Myr}$ for models with $x_{m}=0.001$ (upper panel) or $x_{m}=0.002$ (lower panel). 


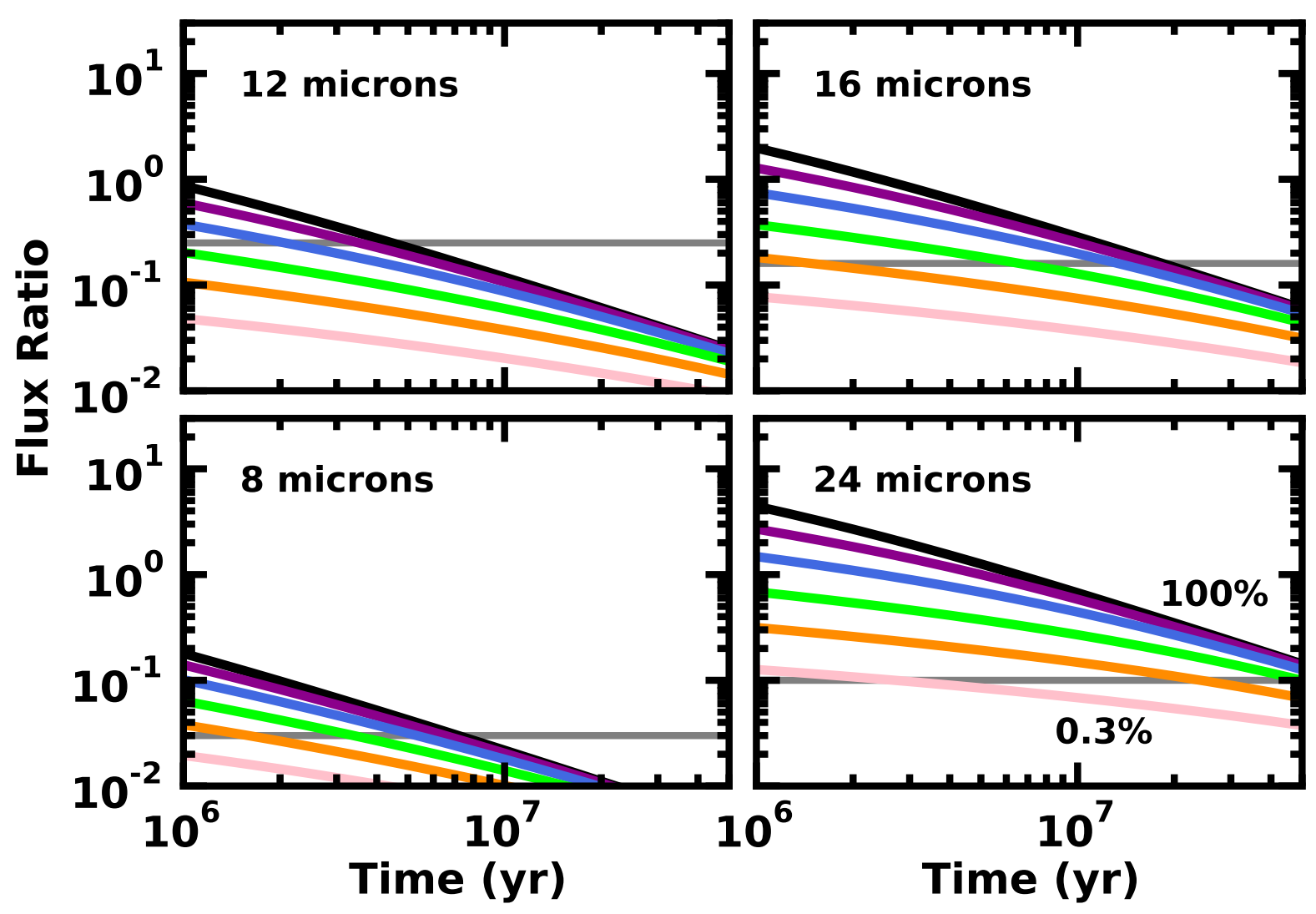

Fig. 4.- Predicted ratio of dust emission $F_{d}$ to photospheric emission $F_{\star}$ in an analytic debris disk model with $r_{\max }=300 \mathrm{~km}$ extending from 0.1 AU to $2 \mathrm{AU}$. For wavelengths of 8-24 $\mu \mathrm{m}$ (as indicated in the legend of each panel), curves plot results for $x_{m}=1.0$ (black curves) to $x_{m}=0.003$ (pink curves) in steps of $\sqrt{10}$. Detecting dust from terrestrial planet formation at 8-12 $\mu \mathrm{m}$ with existing facilities requires conversion of at least $30 \%$ of a MMSN into debris. At $16-24 \mu \mathrm{m}$, as little as $0.3 \%$ of the MMSN is detectable around stars with ages of 5-20 Myr. 


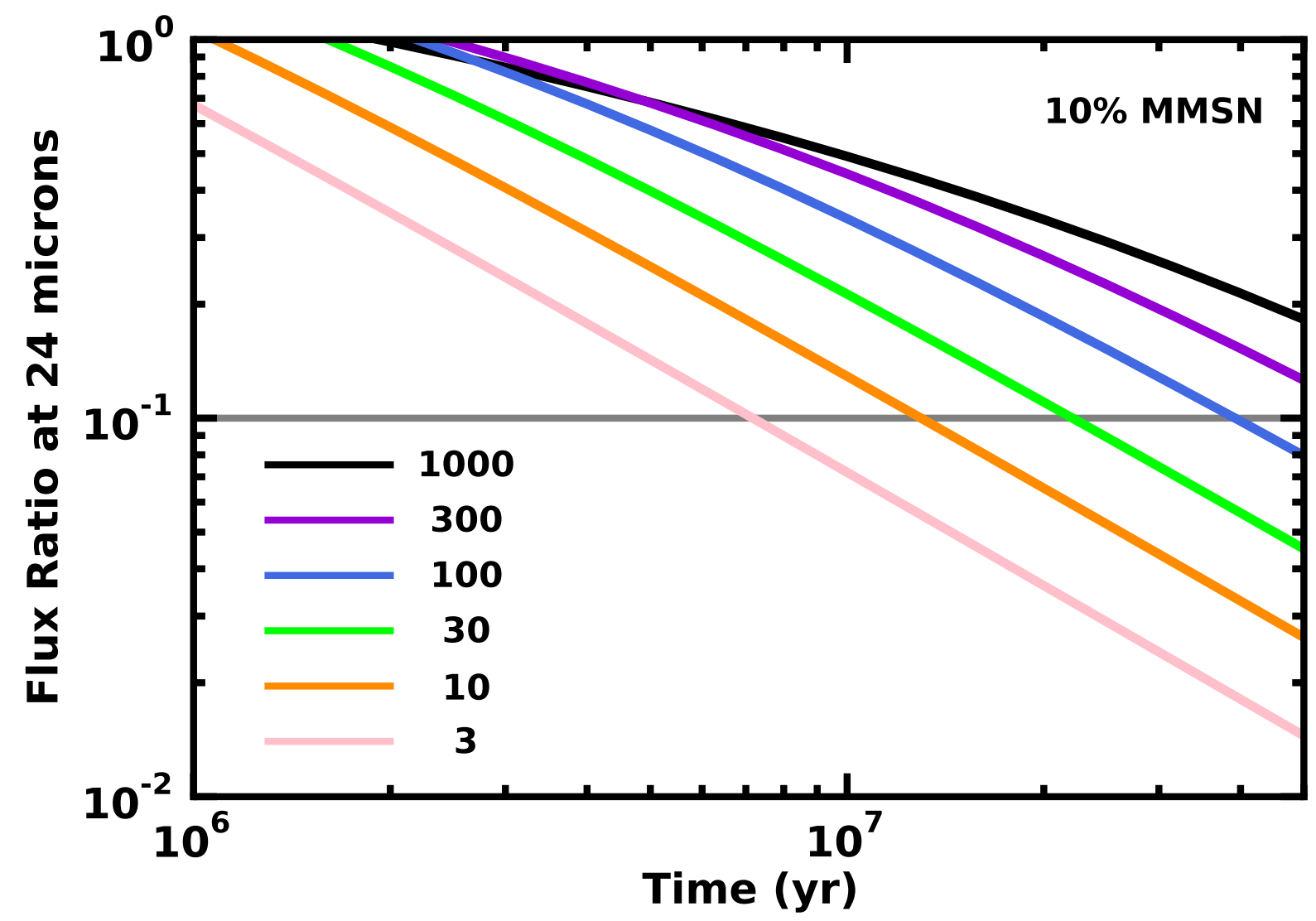

Fig. 5.- As in Fig. 4 for $24 \mu \mathrm{m}$ emission from disks with $x_{m}=0.1$ and $r_{\max }=3-1000 \mathrm{~km}$ as indicated in the legend. At $10 \mathrm{Myr}(20 \mathrm{Myr})$, disks with $x_{m}=0.1$ and $r_{\max } \approx 10-1000 \mathrm{~km}$ $\left(r_{\max } \approx 30-1000 \mathrm{~km}\right)$ produce detectable amounts of $24 \mu \mathrm{m}$ emission. 


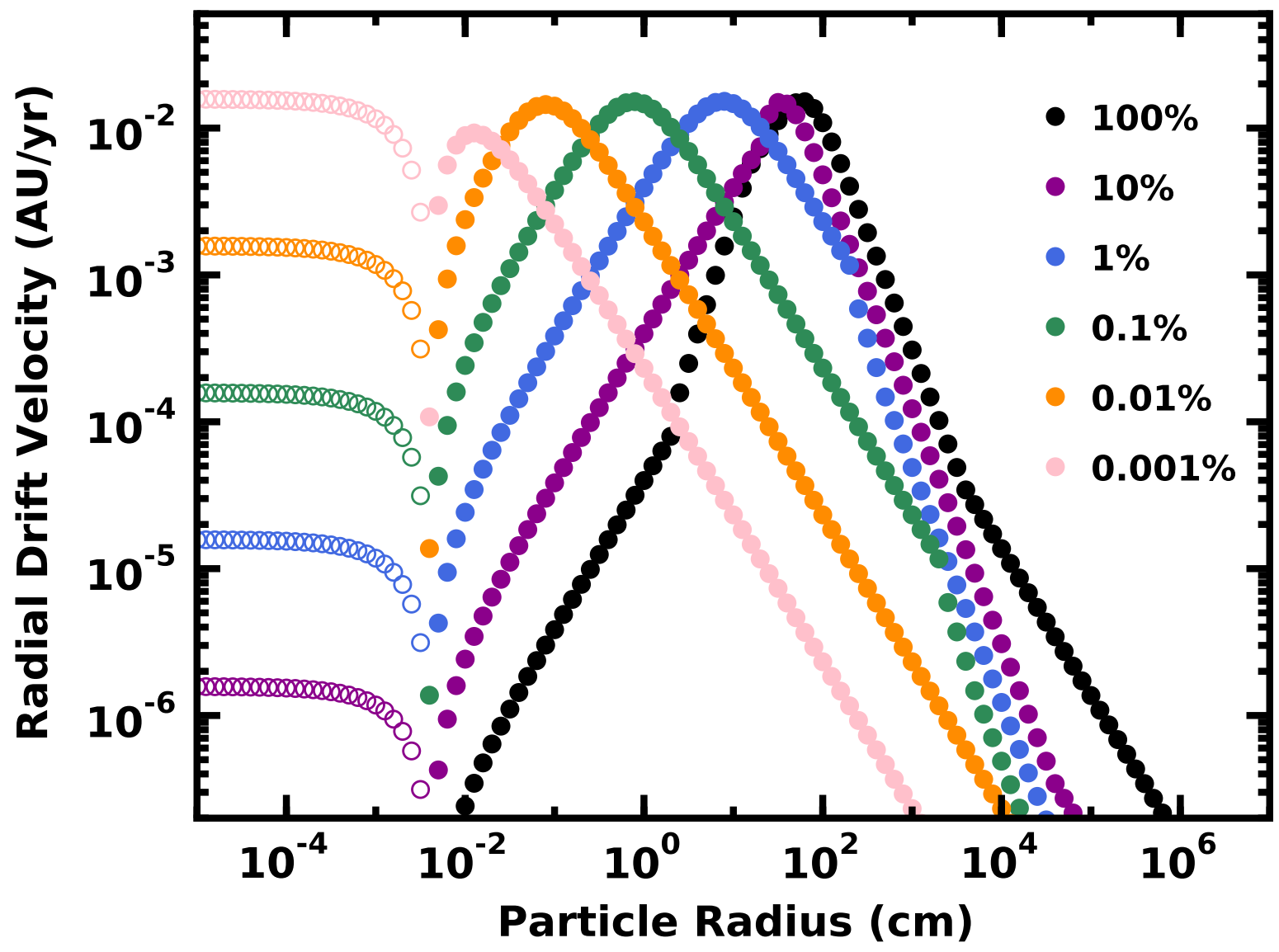

Fig. 6. - Radial drift velocity of particles at $1 \mathrm{AU}$ in a gaseous disk with $\Sigma_{0}=2000 \mathrm{~g} \mathrm{~cm}^{-2}$, $T_{0}=278 \mathrm{~K}, p=1, q=0.5, H_{0}=0.03, s=1 / 8, \gamma=1.4$, and $\mu=2.0$. Filled (open) circles indicate inward (outward) drift. The legend indicates the local surface density relative to the MMSN. At the maximum drift velocity for particles with $\tau_{s} \approx 1,50-100 \mathrm{yr}$ lifetimes are shorter than collisional lifetimes of $1000 \mathrm{yr}$ for debris disks with $L_{d} / L_{\star} \approx 10^{-4}$. When the surface density is less than roughly $0.003 \%$ of the MMSN, lifetimes for very small particles are also shorter than the collisional lifetime. 


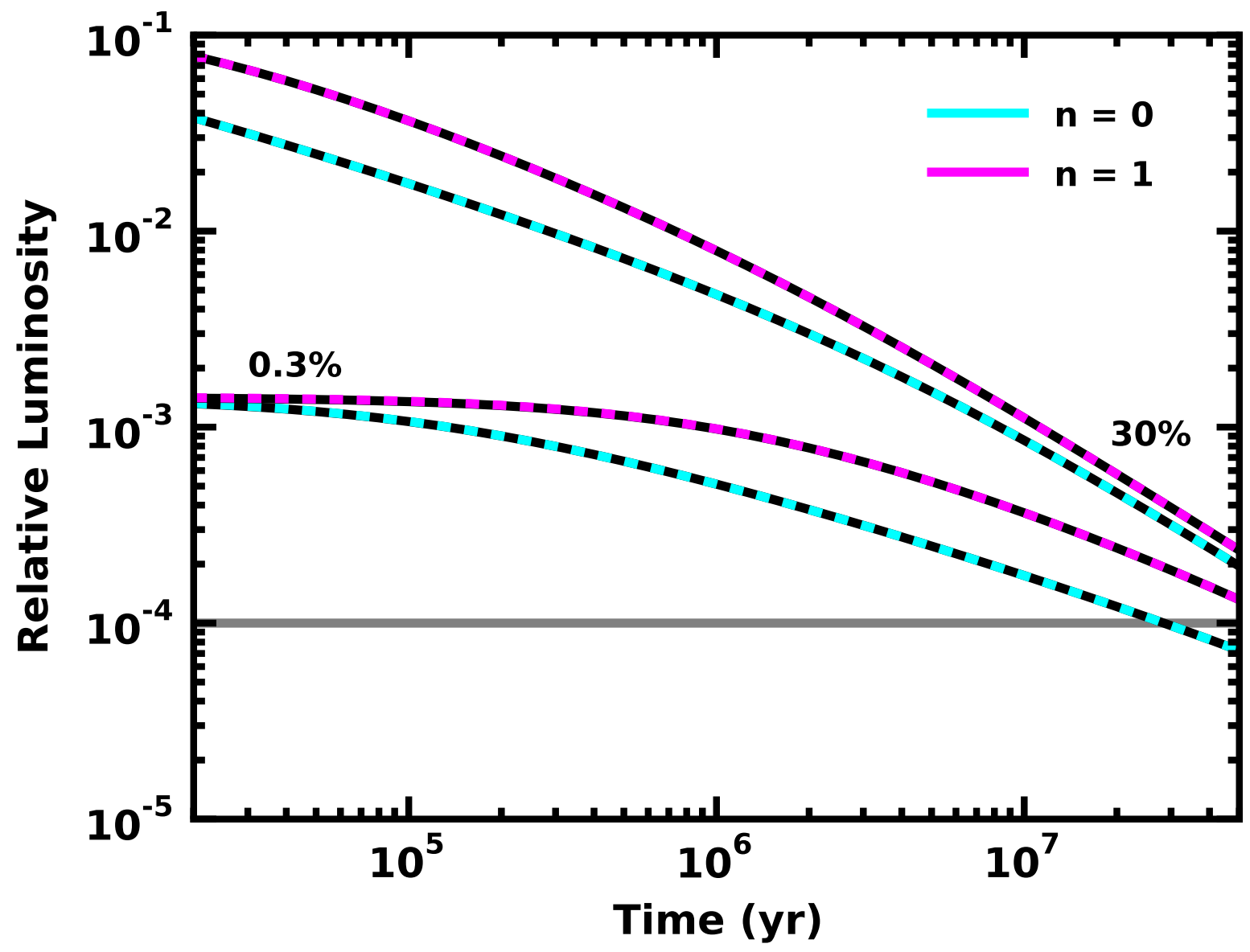

Fig. 7.- Comparison of numerical (dashed black lines) and analytical (dashed cyan or magenta lines) results for the time evolution of debris with $r_{\min }=1 \mu \mathrm{m}, r_{\max }=300 \mathrm{~km}$ in a disk with $x_{m}=0.3$ (upper pair of curves) and $x_{m}=0.003$ (lower pair of curves) extending from $0.1 \mathrm{AU}$ to $2 \mathrm{AU}$. The legend indicates the value of $n$ in the expression $\alpha=\alpha_{0}\left(v^{2} / Q_{D}^{\star}\right)^{-n}$ with $\alpha_{0}=1$. The numerical solution matches the analytic solution to better than $1 \%$ at $t \lesssim$ 1-10 Myr and to better than $0.1 \%$ at $t \gtrsim 10 \mathrm{Myr}$. The grey horizontal line establishes an approximate lower limit on the luminosity detectable with modern instruments. 


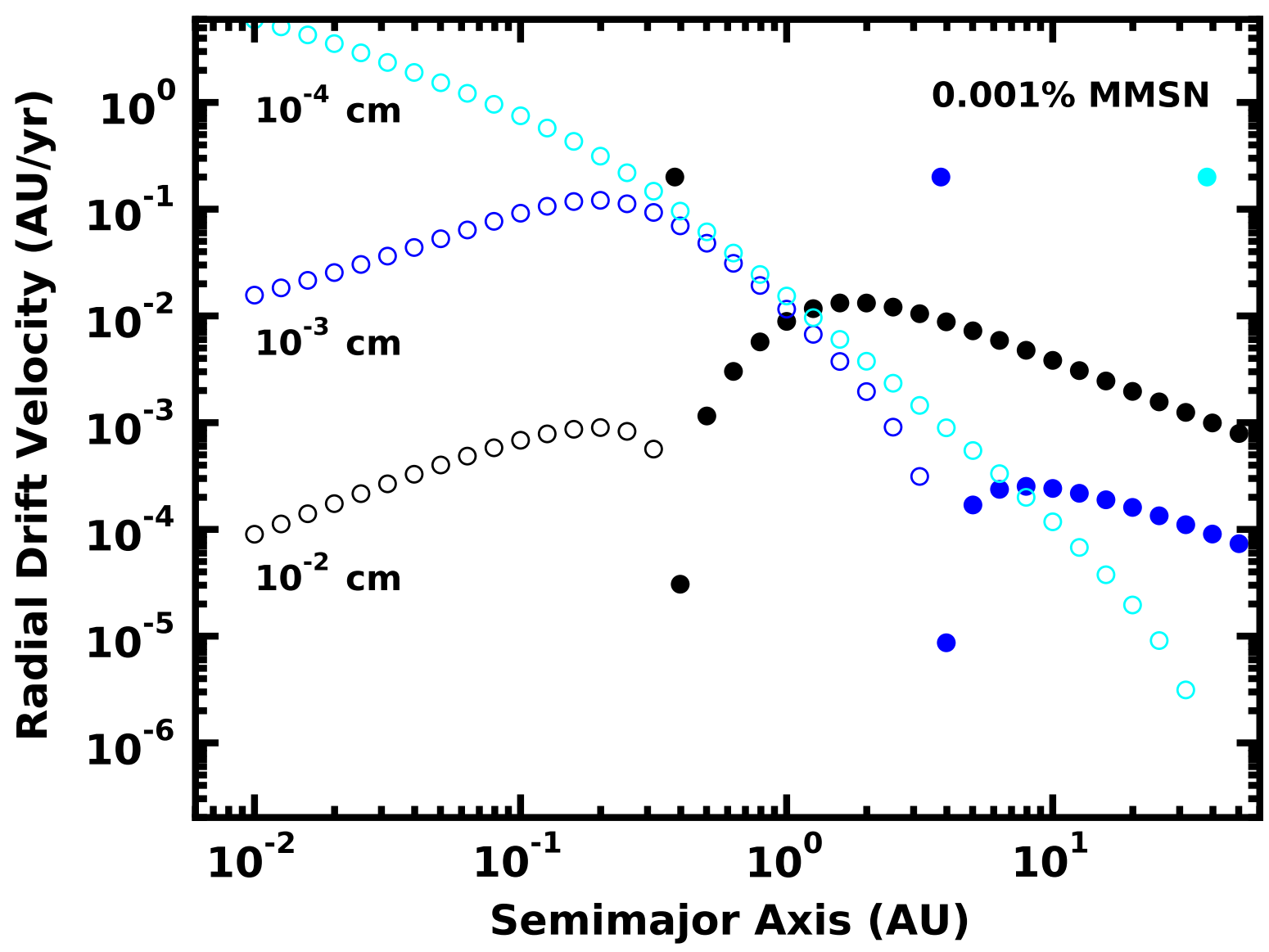

Fig. 8. - As in Fig. 6 for the drift of 1, 10, and $100 \mu \mathrm{m}$ particles as a function of distance from the central star. 